\title{
A Novel Model on Curve Fitting and Particle Swarm Optimization for Vertical Handover in Heterogeneous Wireless Networks
}

\author{
Shidrokh Goudarzi, ${ }^{1}$ Wan Haslina Hassan, ${ }^{1}$ Mohammad Hossein Anisi, ${ }^{2}$ \\ Seyed Ahmad Soleymani, ${ }^{3}$ and Parvaneh Shabanzadeh ${ }^{4}$ \\ ${ }^{1}$ Communication System and Network (iKohza) Research Group, Malaysia-Japan International Institute of Technology (MJIIT), \\ Universiti Teknologi Malaysia, Jalan Semarak, 54100 Kuala Lumpur, Malaysia \\ ${ }^{2}$ Department of Computer System \& Technology, Faculty of Computer Science \& Information Technology, University of Malaya, \\ 50603 Kuala Lumpur, Malaysia \\ ${ }^{3}$ Faculty of Computing, Universiti Teknologi Malaysia, UTM Johor Bahru, 81310 Johor, Malaysia \\ ${ }^{4}$ Centre for Artificial Intelligence and Robotics, Universiti Teknologi Malaysia, 54100 Kuala Lumpur, Malaysia
}

Correspondence should be addressed to Mohammad Hossein Anisi; anisi@um.edu.my

Received 18 June 2015; Accepted 6 September 2015

Academic Editor: Yihua Lan

Copyright (C) 2015 Shidrokh Goudarzi et al. This is an open access article distributed under the Creative Commons Attribution License, which permits unrestricted use, distribution, and reproduction in any medium, provided the original work is properly cited.

\begin{abstract}
The vertical handover mechanism is an essential issue in the heterogeneous wireless environments where selection of an efficient network that provides seamless connectivity involves complex scenarios. This study uses two modules that utilize the particle swarm optimization (PSO) algorithm to predict and make an intelligent vertical handover decision. In this paper, we predict the received signal strength indicator parameter using the curve fitting based particle swarm optimization (CF-PSO) and the RBF neural networks. The results of the proposed methodology compare the predictive capabilities in terms of coefficient determination $\left(R^{2}\right)$ and mean square error (MSE) based on the validation dataset. The results show that the effect of the model based on the CF-PSO is better than that of the model based on the RBF neural network in predicting the received signal strength indicator situation. In addition, we present a novel network selection algorithm to select the best candidate access point among the various access technologies based on the PSO. Simulation results indicate that using CF-PSO algorithm can decrease the number of unnecessary handovers and prevent the "Ping-Pong" effect. Moreover, it is demonstrated that the multiobjective particle swarm optimization based method finds an optimal network selection in a heterogeneous wireless environment.
\end{abstract}

\section{Introduction}

An efficient algorithm to determine the best network among the available ones is significant for wireless networks. The merits of each available network should be realized to discover the best network. Several multicriteria schemes based on artificial intelligence methods such as fuzzy logic, neural networks, and genetic algorithms by $[1,2]$ have weaknesses on scalability and modularity issues. They simply cannot manage based on the high numbers of radio access technologies (RATs) and the criteria of the heterogeneous wireless networks (HWN). These algorithms have several weaknesses in terms of scalability and complexity because they enter all inputs from the different RATs to one fuzzy logic block simultaneously rather than an exponential increase based on the number of inference rules.

The field of future wireless networks is one of the most attractive areas among researchers [3-6]. The proposed algorithms in this area of research are classified into different groups based on the analyses, studies, and tutorials found in the related literature [3-6]. These algorithms are classified into different groups based on the expended decision technique. Reference [7] proposed a novel method for RAT selection, namely, the hopfield neural network RAT selection mechanism (HRM), that utilizes the hopfield neural networks as a strong decision-making tool. A new approach using 
information about data rate, monetary cost, and received signal strength as different parameters to make a handover decision has been reported by $[8,9]$. The main weaknesses are related to the computation of the error function and the Jacobian inversion for acquiring a matrix in which the sizes are equal to the whole of all the weights in the neural network (NN). Therefore, the necessity for memory is very high. Existing algorithms $[8,9]$ consider the service fee, the received signal strength information (RSSI), user preference, and so forth. The proposed algorithm in comparison with the traditional RSSI-based algorithm enhances the outcomes mainly for both the user and the network as a result of the offered fuzzy based handover systems. In terms of hybrid categories, [10] proposed a PSO-FNN-based vertical handover decision scheme that could make an intelligent handover decision based on the analysis of the network's position. The authors of [11] mainly dealt with a novel vertical handover decision algorithm built on fuzzy logic with the assistance of the Grey theory and the dynamic weights adaptation. A neurofuzzy multiparameter-based vertical handover decision algorithm (VHDA) was proposed by [12]. The results of the performance evaluation carried out by the handover quality indicator (used to quantify QoS), which is related to the "Ping-Pong" effect, ESA, and throughput, proved that the proposed VHDA offered better QoS than the existing vertical handover methods. Pahlavan et al. [13] method is a good representation of applying a fuzzy logic-based normalized quantitative decision algorithm and a differential prediction algorithm with a high level of accuracy.

The vertical handover schemes stated above have their own benefits, but they do not consider the complexity of the network selection, and the allowance of lower computation cost function is unreasonable. Clearly, the decision process should focus on a steadfast, intelligent algorithm to execute an accurate decision and to shift to the best network candidate quickly. The goal of this study is to propose a novel network selection optimization algorithm that takes advantage of the prediction model using the CF-PSO to meet the requirements stated above. The usage of the CFPSO algorithm for prediction with the PSO algorithm for selection has three purposes: (1) to serve as a validation algorithm for the outcome of the MOPSO, (2) to decrease the number of unnecessary handovers and prevent the "PingPong" effect, and (3) to select the best candidate access point among various access technologies. This paper provides a comparison based on two prediction methods, namely, the CF-PSO and the RBF neural network, to predict the RSSI. More importantly, this comparison evaluates the two methods from different aspects, for example, time, coefficient determination, and mean squared error. The results show that the CF-PSO has better performance, which will be presented in-depth in the following. The proposed method is a step towards future computer-based optimization methods where huge uncertainties by the optimization algorithm must be avoided. To do this, the prediction algorithm is combined with the particle swarm optimization. The proposed MOPSO-based vertical handover decision algorithm can make an intelligent handover decision based on the network

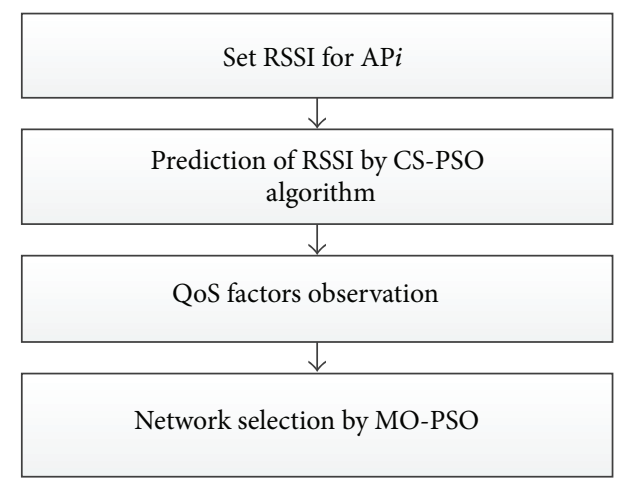

FIGURE 1: Proposed network selection model based on the Multiobjective Particle Swarm Optimization (MOPSO) in a wireless heterogeneous environment.

position. The proposed network selection model is presented in Figure 1.

The outline of this paper is as follows. First, the related works are described in Section 2. In Section 3, the proposed prediction methods based on CF-PSO and RBF network are introduced. Then, the results of comparison based on the proposed models are illustrated. Section 4 illustrates the MOPSO-based vertical handover decision algorithm. Section 5 analyzes the performance of the algorithm through the simulation results. Finally, Section 6 concludes the paper.

\section{Related Work}

Many network selection algorithms have been proposed in literature, in which PSO has been used for handover decisions. It is initialized with a set of random particles (solutions) that finds an optimal result by updating the generations. The optimum results are called particles, which fly throughout the problem space by following the current optimal particles. In addition, a GSM-like [14] hard handover algorithm has been proposed where countermeasures prevent the PingPong effect by providing a baseline handover threshold against power fluctuations due to channel variations. The authors defined four rules where two rules were for preventing unnecessary handovers (Ping-Pong effects) and one rule was aimed at helping the loaded eNB $\mathrm{j}$ by delaying handovers from eNB i. Moreover, the other rule was aimed at alleviating the loaded eNB i by advancing handovers towards eNB j. The PSO algorithm denoted that the neighbor with which the eNB has the largest handover traffic exchange was the best neighbor. Prior knowledge is required on the optimization process that provided the parameterized form of the controller, which was optimized by the MOPSO. It has been shown that the dynamic optimization outperformed the static optimization and produced a better mobility load balancing self-organizing network controller, which improved the throughput and access probability by a few percents with respect to the planning solution.

Wang et al. [15] proposed an always best connected (ABC) maintained QoS handover decision scheme based on the niche PSO algorithm. This literature reported the access 
network in terms of its current load and considered the terminal in terms of its current velocity and residual electric capacity. The authors also studied the application of QoS requirement, preference of the user over the access network coding system, and preference of the user over the access network provider, and so forth.

In addition, Venkatachalaiah et al. [16] presented a technique for forecasting the signal strength value that aids in offering efficient handovers in wireless networks and the PSO was expended to fine-tune the weighting function of the handover decision. The proposed technique minimized the number of handovers and was shown to have a very short calculation time and better prediction accuracy compared to hysteresis based decisions. In this paper, they described the use of the Grey model in combination with the fuzzy logic and PSO algorithms. Since prediction error is inevitable, the output from the Grey model could be compensated with the use of a fuzzy controller and then fine-tuned using PSO algorithms.

The method by Liu and Jiang [11] is a good symbolic example of using a fuzzy logic-based normalized quantitative decision algorithm and a differential prediction algorithm with a high level of accuracy. This scheme tried to control handovers between WLANs and UMTS. A predecision section was used in this algorithm. The forward differential prediction algorithm was used to acquire the predictive RSS, which could trigger a handover in advance. Moreover, the predecision method was applied before the handover decision module, which is able to filter the unnecessary data and to provide a correct handover trigger. Optimizing the selection method is an important subject of research, which can lead to the decline of network signaling and mobile device power loss and also boost network QoS and grade of service (GoS).

The main focus of this paper is to achieve better QoS while selecting the ABC network, using a novel RSSI prediction algorithm based on curve fitting which uses an enhanced PSO and a network selection algorithm based on MOPSO for vertical handover in heterogeneous wireless networks. The network selection algorithm utilizes dynamic optimized weights using the AHP method to select the $\mathrm{ABC}$ network in the heterogeneous environment of the cellular networks. Dynamic weights of the parameters used in the cost function for network selection are optimized using an improved MOPSO algorithm.

\section{Prediction of RSSI Using the Proposed Prediction Methods}

In this section, we intend to show that the CF-PSO has better performance compared to the RBF as a prediction model. Received signal strength indicator or RSSI is a common metric used in handover decision-making [17]. The quality and distance for each access point (AP) in the range can be analyzed by scanning the RSSI via a mobile node (MN). When the MN changes its location to an AP, the level of RSSI for that AP will change. A real-tested measurement is used to realize the RSSI level through the MN movement. This

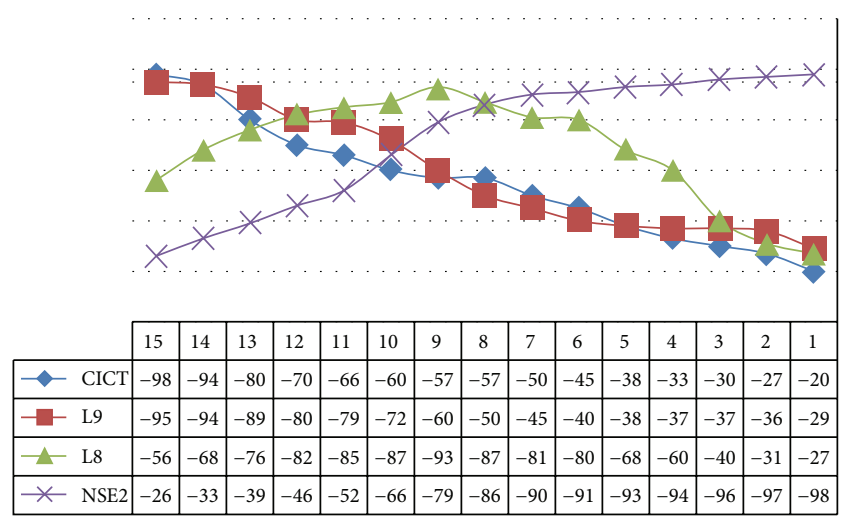

FIGURE 2: Collected RSSI for four APs through the scanning phase by the Chanalyzer software.

scenario comprises one laptop as the MN within the APs used to offer the UMTS and WLAN services. Data is collected via a MetaGeek Wi-Spy spectrum analyzer and the Chanalyzer software. The most necessary L2 information was collected using the Chanalyzer software as the information assists in scheming the proposed prediction algorithm. Figure 2 displays the varying RSSI values during the MN movement during $15 \mathrm{Sec}$. The RSSI is gathered by the Chanalyzer software, which is installed in one laptop moving across the four APs.

3.1. Prediction Using the Curve Fitting-Based Particle Swarm Optimization. A prediction technique for vertical handover using the CF-PSO algorithm is presented in this section. Curve fitting is based on the procedure of creating a curve or mathematical function, which has the best fit to the data points, subject to constraints. In addition, the PSO is a kind of swarm intelligence, which utilizes a group of particles that form a swarm, moving across the search space observing the best solution. Particles are treated as a point in an $\mathrm{N}$ dimensional space, which modifies their "flying" based on their own flying experience as well as the flying experience of other particles. Particles can change their positions based on various types of information, namely, existing positions, recent velocities, the distance between the current position and the personal best position (pbest), and the distance between the current position and the global best position (gbest). Particles can route their positions in the solution space, which are related to the best solution (fitness) that has been realized thus far by these particles. This value is called the personal best, pbest. Another best value, which is tracked by the PSO, is the best value distance obtained subsequently by the particles in the region of these particles. This item is called gbest. The basic concept of the PSO focuses on the fasttracking particles based on their pbest and gbest locations each time. The searching concept of the PSO algorithm is shown in Figure 3. The change of the particle's position can be mathematically formed based on the following:

$$
\begin{aligned}
V_{i}^{m+1}= & \omega * V_{i}^{m}+c_{1} \text { rand }_{1} \times\left(\text { pbest }_{i}-s_{i}^{m}\right)+c_{2} \text { rand }_{2} \\
& \times\left(\text { gbest }-s_{i}^{m}\right),
\end{aligned}
$$




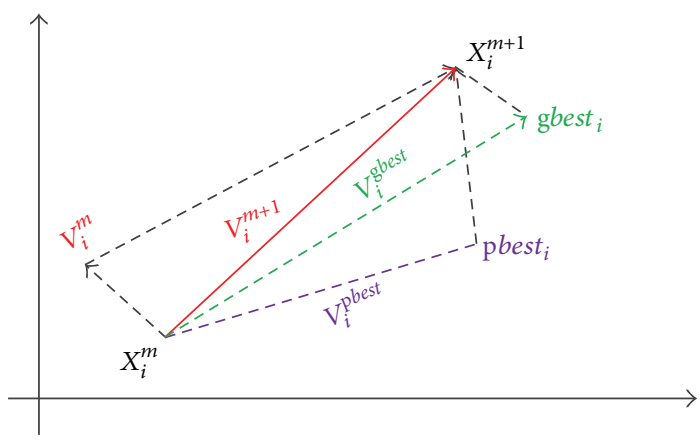

FIgURE 3: Searching concept of the PSO.

where $V_{i}^{m}$ is velocity of particle $i$ in iteration $m, \omega$ is inertia factor, $c_{i}$ is weighting factor, rand is uniformly distributed random number between 0 and $1, s_{i}^{m}$ is current position of particle $i$ in iteration $m$, pbest ${ }_{i}$ is pbest of particle $i$, and gbest is gbest of the group.

A small inertia weight facilitates a local search and a large inertia weight $(\omega)$ assists a global search. Linearly declining the inertia weight from a large value to a small value through the course of the PSO run provides the best PSO performance in comparison to the stable inertia weight settings. Inertia factor $(\omega)$ is increased by the velocity of a particle at $n$th position to achieve the changed velocity update as shown in (1). $\omega$ is initialized to 1.0 and is steadily decreased over time. The CF-PSO program was created and executed in the MATLAB.

In the scanning stage, the RSSI data for each AP will be entered as the input values to the CF-PSO to compute each point of data (RSSI). The process is done by building upper and lower bounds covering each RSSI value point collected for the period of scanning time. Thus, the CF-PSO achieves the next predicted RSSI value depending on how far the real RSSI point is from the curve of fitting. This permits the MN to forecast the next RSSI value of available APs and its current attached AP and then obtains a handover decision with a low latency.

The RSSI data for each AP will be entered as the input values to the CF-PSO to compute each point of data throughout the scanning step. Creating higher and lower limits covering each RSSI value point collected during the scanning time completes the procedure. Therefore, the CF-PSO gets the next predicted RSSI rate depending on the distance of the real data with the curve of fitting. Through this method, the MN can predict the next RSSI value of the available APs and the existing attached AP to the available AP. Then, it makes the best handover decision with the lowest latency.

In this study, the polynomial model has been selected for use in the curve fitting model to obtain the degree of fitness for each set of RSSI data that is related to a specific AP. The polynomial model has enough flexibility for data with a high level of complexity, such as the RSSI data. In addition, it is linear which shows that the fitting process applies in plain and effective ways. The CF-PSO works on collecting the RSSI for each AP to find a close fit between the points of RSSI values and then obtains the expected curve between them. In other

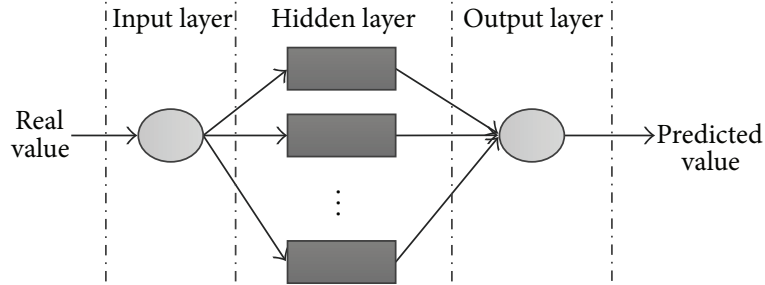

FIGURE 4: RBF neural network structure.

words, the curve fitting usually works to get close values to maintain the curve in the production region. Equation (2) presents the Polynomial model in the CF-PSO:

$$
F(x)=\left[P_{1} X^{4}+P_{2} X^{3}+P_{3} X^{2}+P_{4} X^{1}+P_{5}\right]
$$

Further details about the RSSI prediction using the CF-PSO are presented in Section 6.

3.2. Prediction Using the RBF Neural Network Method. The artificial neural network (ANN) is used to develop, optimize, estimate, predict, and monitor complicated systems. A new and effective feed forward neural network with three layers called the RBF neural network has fine characteristics of approximation performance and global optimum [18]. In general, the RBF network consists of the input layer, the hidden layer, and the output layer. Each neuron in the input layer is responsible for transferring the recorded signal to the hidden layer. In the hidden layer, the radial basis function is often used as the transfer function, while a simple linear function is usually adopted in the output layer. The RBF program was implemented in the MATLAB. The RBF neural network with three layers as used in the paper is displayed in Figure 4.

3.3. Comparative Analysis. After the scanning step, the RSSI data of each AP will be inserted into the CF-PSO as the main inputs for evaluating the best prediction achieved from the RSSI variations throughout the time of the MN's movement. Hence, the MN will acquire an exact method that firstly analyzes and then expects the next incoming RSSI for APs in the scanning region.

In order to assess the performance of fit in the CFPSO as shown in Figure 5, the residual analysis has been changed and used. This is to justify the ways in which the CF-PSO can predict new RSSI values, with a great degree of certainty, resulting from extremely variable RSSI data collected from the APs. Three statistical estimators, namely, the mean squared error (MSE) in (3), the coefficient of determination $\left(R^{2}\right)$ in $(4)$, and the root mean square error (RMSE) in (5), where if the RMSE is zero then the method has outstanding performance, were used to evaluate the performance of the CF-PSO: 


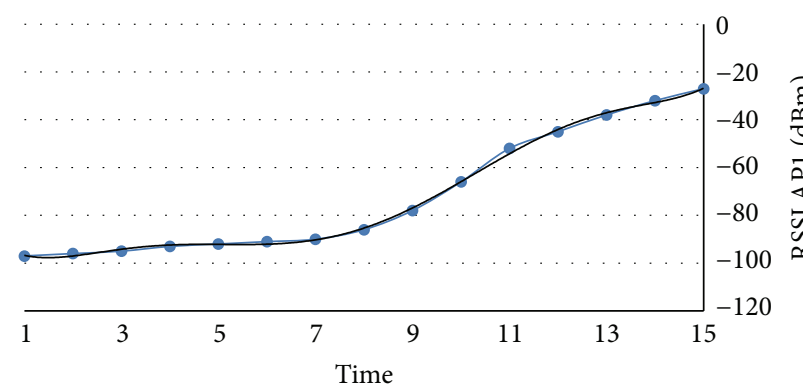

RSSI AP1 versus time Fitted curve AP1

(a) The prediction bound for AP1 RSSI

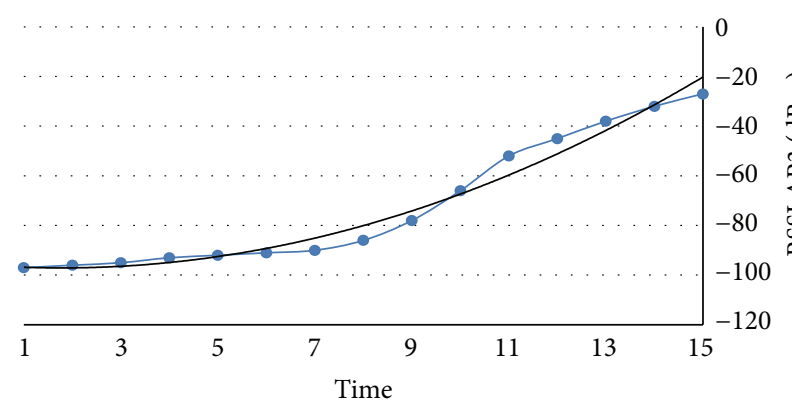

RSSI AP3 versus time Fitted curve AP3

(c) The prediction bound for AP3 RSSI

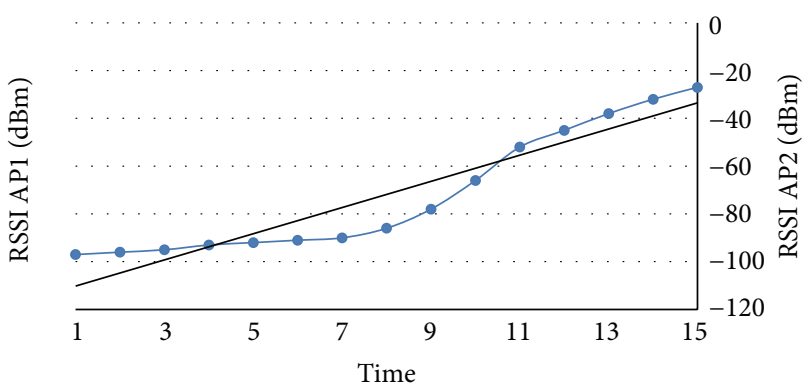

RSSI AP2 versus time - Fitted curve AP2

(b) The prediction bound for AP2 RSSI

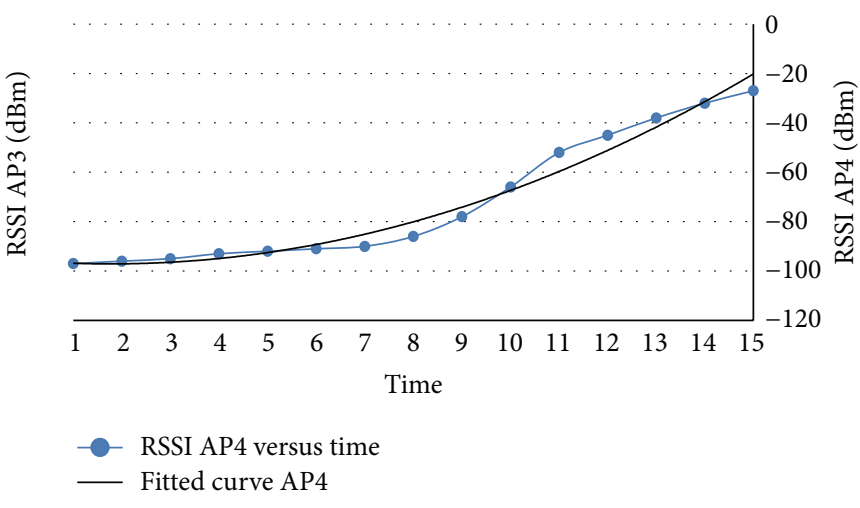

(d) The prediction bound for AP4 RSSI

FIgure 5: Process of CF-PSO prediction for RSSI of AP1, AP2, AP3, and AP4.

$$
\begin{aligned}
\text { MSE } & =\frac{1}{r \sum_{i=1}^{r}\left(v_{p i}-v_{a i}\right)^{2}} \\
R^{2} & =1-\frac{\sum_{i=1}^{r}\left(v_{p i}-v_{a i}\right)^{2}}{\sum_{i=1}^{r}\left(v_{p i}-v_{a v}\right)^{2}} \\
\text { RMSE } & =\sqrt{\frac{1}{r \sum_{i=1}^{r}\left(v_{p i}-v_{a i}\right)^{2}}},
\end{aligned}
$$

where $r$ is the number of points, $v_{p i}$ is the estimated value, $v_{a i}$ is the actual value, and $v_{a v}$ is the average of the actual values. The coefficient of determination, $R^{2}$, of the linear regression line between the estimated values of the neural network model and the required output was also used as a measure of performance [19]. The closer the $R^{2}$ value is to 1 , the better the model fits to the actual data [20]. This measurement processes how successful the fit is in describing the change in the data. In other words, $R$-square is the square of the correlation between the response values and the predicted response values. It is also named the square of the multiple correlation coefficients and the coefficient of multiple determinations. $R^{2}$ 's for AP1, AP2, AP3, and AP4 are 0.9973, 0.9818, 0.99278, and 0.9938 , respectively. The correlation coefficient squared $\left(R^{2}\right)$ in four sets of calibration curves ranges from 0.98 to
1.00 indicating an almost perfect linearity of all the curves. Figure 6 shows further details of AP1, AP2, AP3, and AP4.

The artificial neural network with RBF was used to estimate the RSSI data. In this section, the explanation of the experiment using the RBF neural network is presented. The RSSI data will be inserted as inputs into the RBF in order to examine the best prediction using this method. The related results for the four APs are stated in Figure 7. In summary, the RBF network consists of three layers including the input layer, the hidden layer, and the output layer. The ANN executes a nominal computation to offer an output. Computation comprises the one-pass arithmetic steps. The iterative and nonlinear computations are not complicated in offering an output. The RBF networks were chosen because this method involves a simple design that has just three layers. In this study, the number of neurons in the hidden layer is set to 15 , the mean squared error (MSE) is 0.1 according to the actual training process, and the $\sigma$ (sigma) parameter is the width of RBF by 0.02 .

The main advantage is that the RBF has a hidden layer that includes nodes known as the RBF units. Each RBF has main factors that designate the location, deviation, or width of the function's center. The hidden component processes the distance from the input data vector and the center of its RBF. If the distance from the specific center to the input data vector is zero, then the RBF has its own peak, and if the distance increases, then the peak of RBF will decline steadily. 

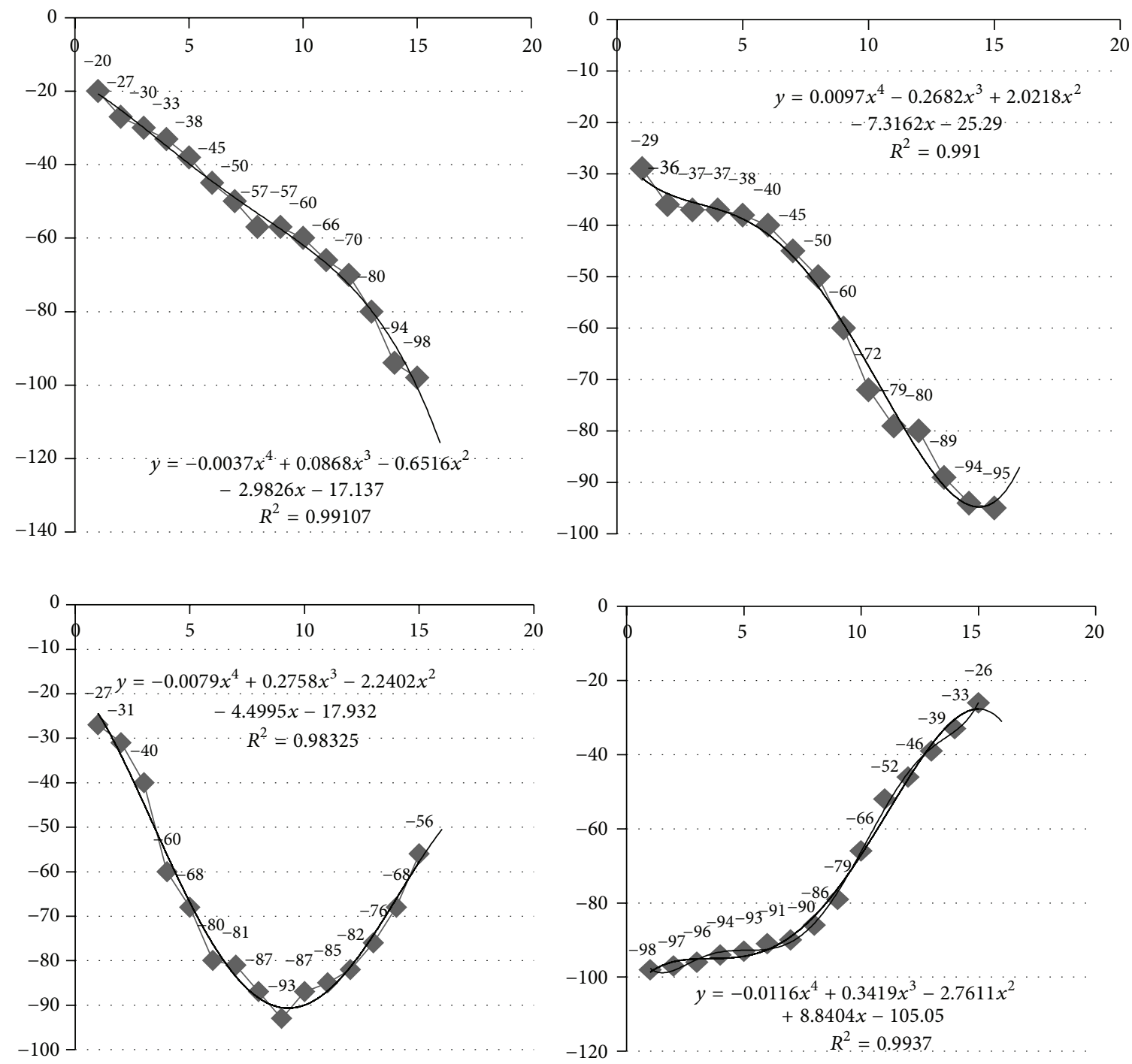

FIGURE 6: $R^{2}$ for AP1, AP2, AP3, and AP4.

In $\mathrm{RBF}$, the hidden layer has different sets of weights that are divided into two sets. These weights can connect the hidden layer to the input and output layers as linkages. The subjects of the basis functions are fixed into the weights that are connected to the input layer. The issues of the network outputs are fixed into the weights that connect the hidden layer to the output layer. Since the hidden units are nonlinear, the outputs of the hidden layer can be merged linearly and, consequently, the processing is fast. The output of the network is retrieved using the following [21]:

$$
y_{k}(x)=\sum_{j=1}^{N} w_{k j} \emptyset_{j}(x)+w_{k 0},
$$

where $N$ is the number of basis functions, $w_{k j}$ represents a weighted connection between the basis function and the output layer, $x$ is the input data vector, and $\emptyset_{j}$ is the nonlinear function of unit $j$, which is typically a Gaussian form as shown in the following [21]:

$$
\emptyset_{j}(x)=\exp \left(-\frac{\|x-\mu\|^{2}}{2 \sigma_{j}^{2}}\right),
$$

where $x$ and $\mu$ are the input and the center of the RBF unit, respectively, and $\sigma_{j}$ is the spread of the Gaussian based function [21]. The weights can be optimized by the least mean square (LMS) algorithm once the centers of the RBF units are determined. The centers are selected randomly or by clustering the algorithms. In this paper, the centers were nominated from the dataset randomly. The radial basis artificial neural network model was trained to minimize the MSE with the parameter (RSSI) as input and the desired output (predicted RSSI). To design and verify the reliability of the proposed model, the dataset was divided into two different sets including the training and test data that are $80 \%$ and $20 \%$ of the total data, respectively. The test data are not presented to the network in the training process. When 

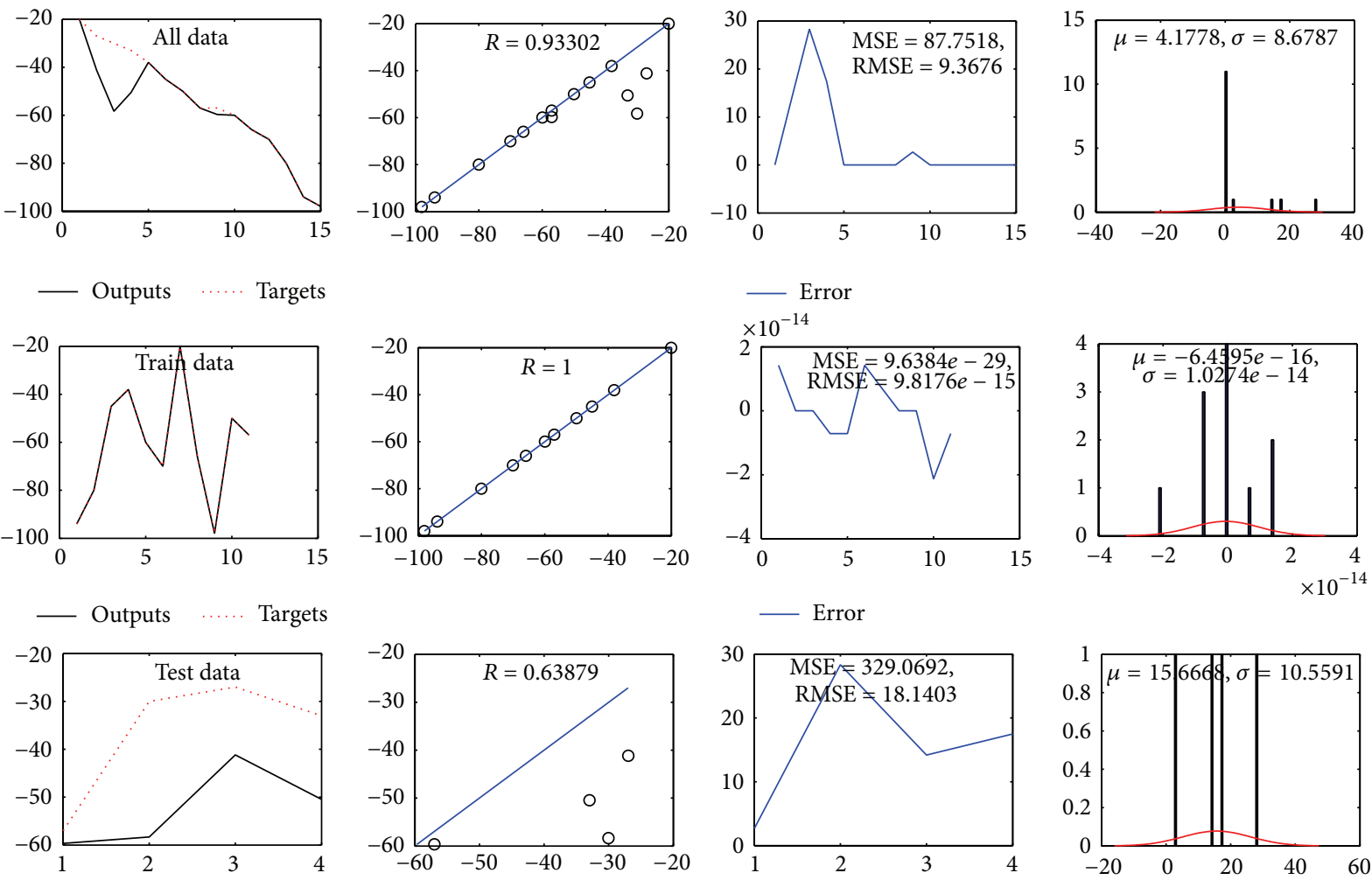

— Outputs ..... Targets

(a) The RBF prediction values for AP1
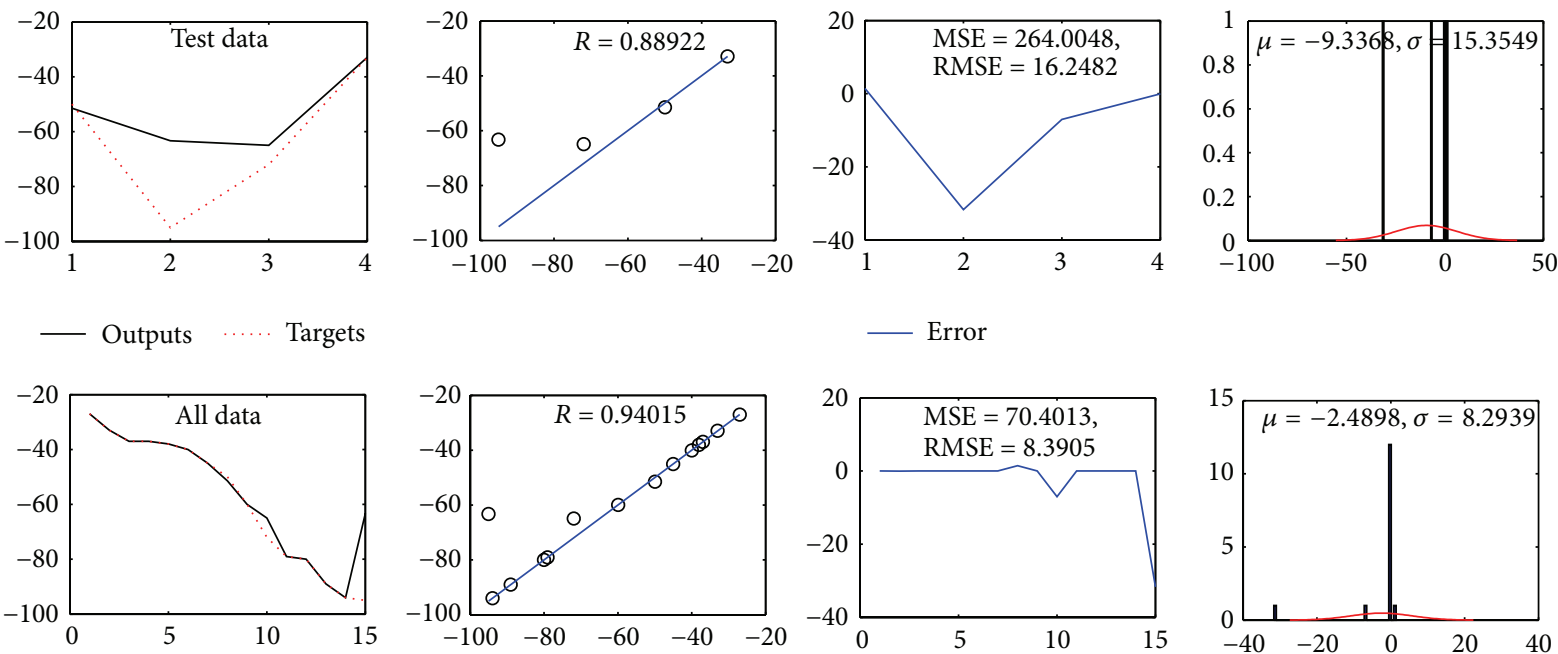

Error

_ Outputs ….. Targets
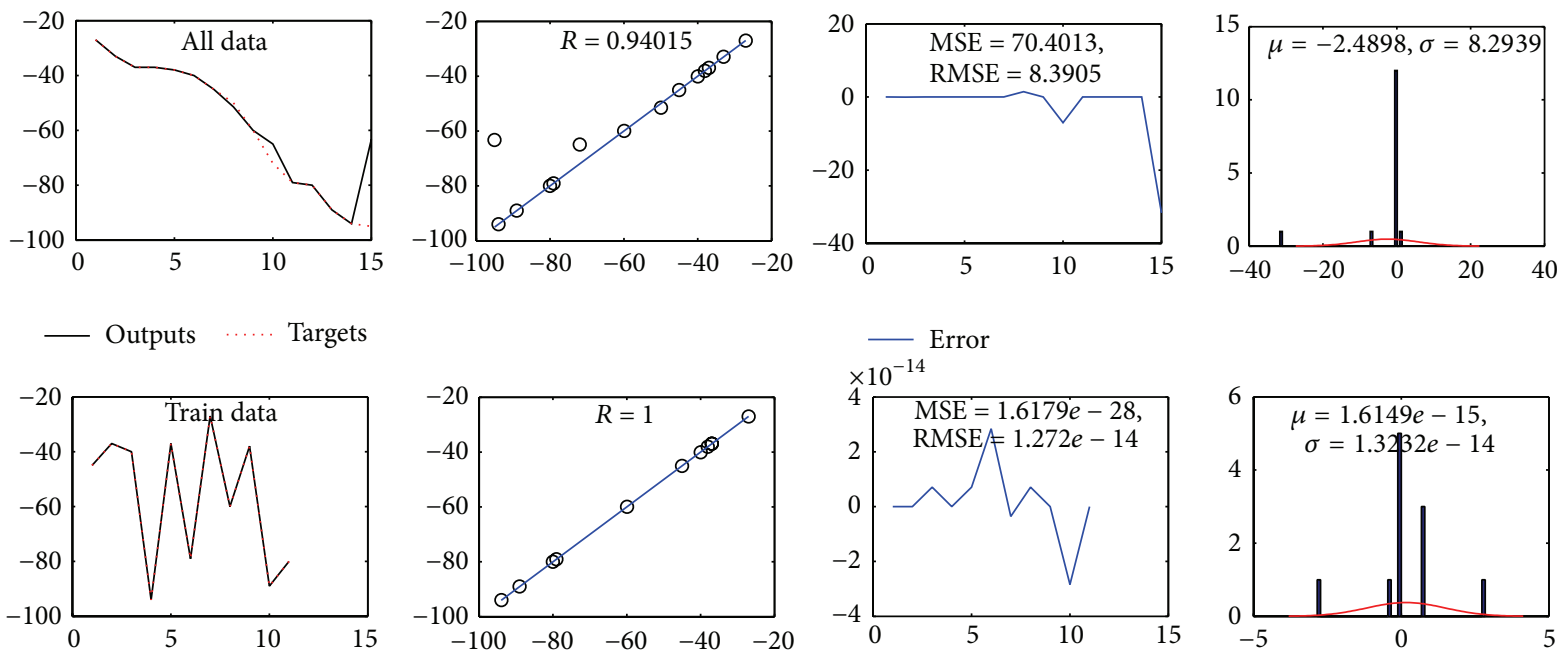

- Outputs ….. Targets

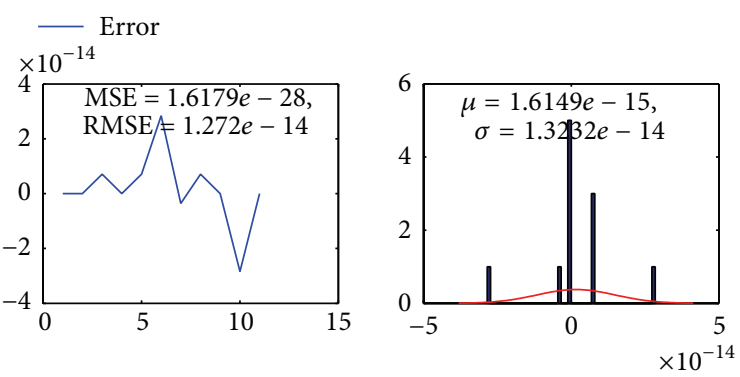

(b) The RBF prediction values for AP2

Figure 7: Continued. 

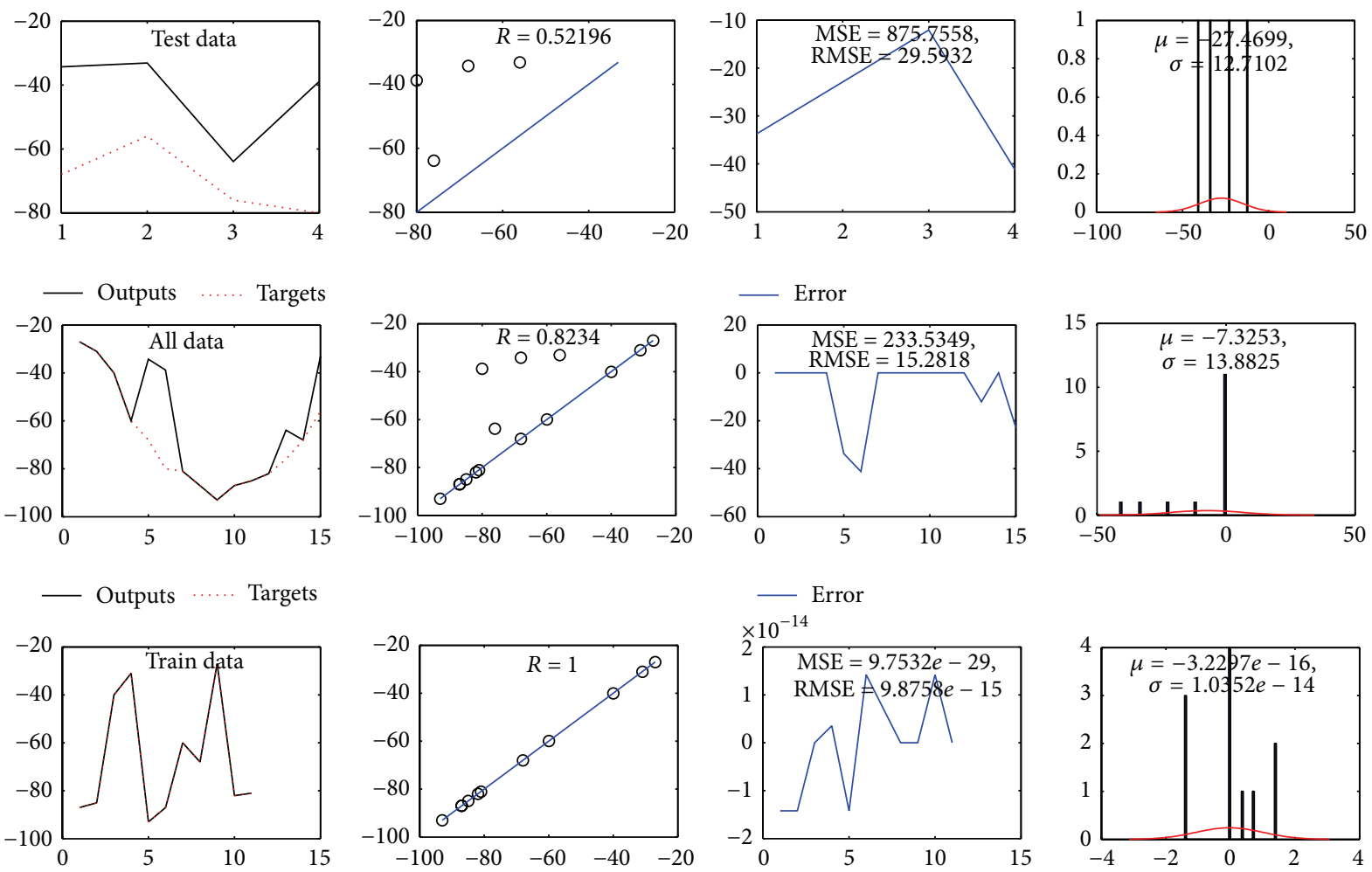

— Outputs …. Targets

— Error

(c) The RBF prediction values for AP3 RSSI
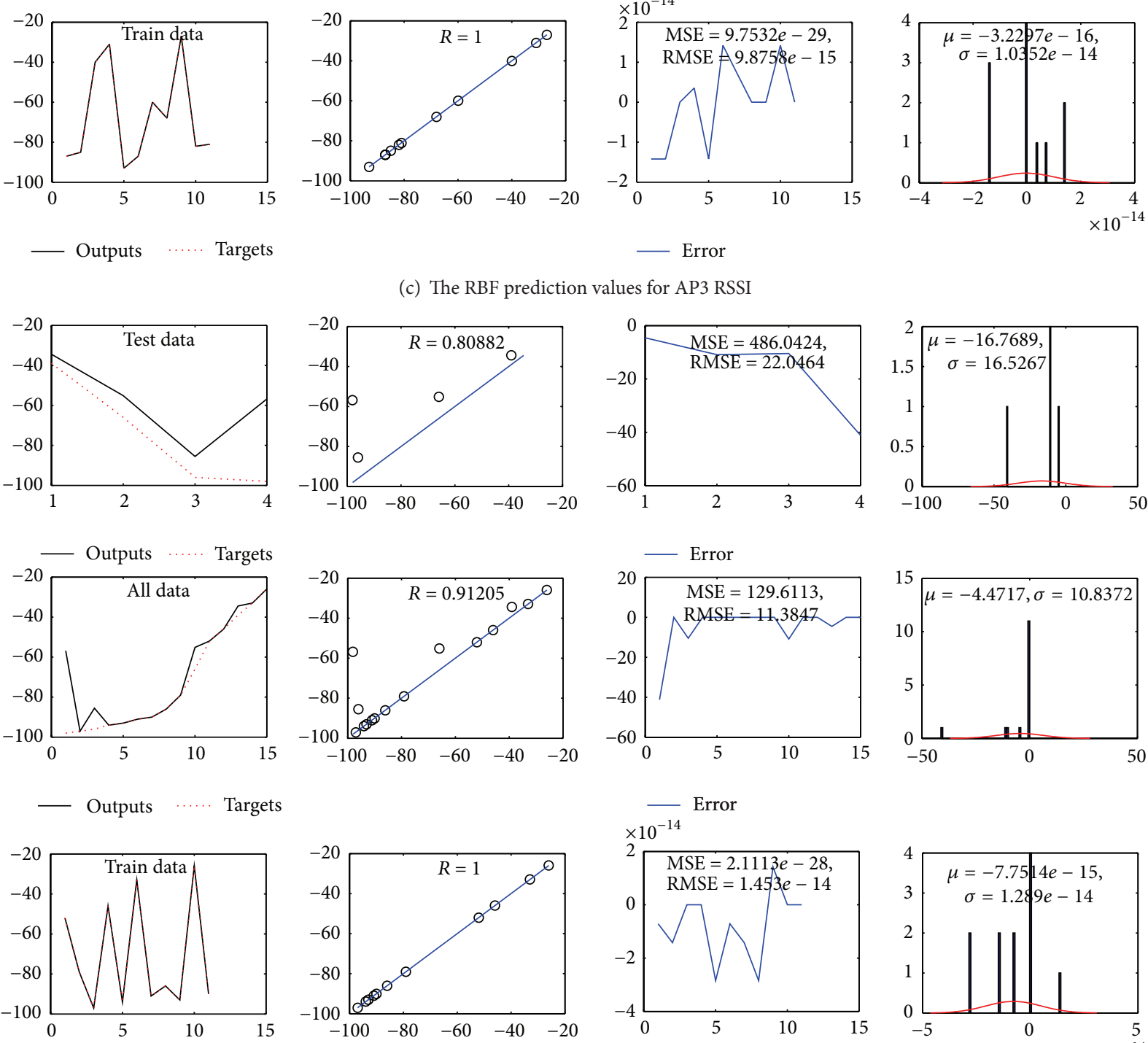

- Outputs ..... Targets

(d) The RBF prediction values for AP4 RSSI

FIgURE 7: Overall performance of RBF for AP1, AP2, AP3, and AP4. 
TABLE 1: Real and predicted RSSI values.

\begin{tabular}{|c|c|c|c|c|c|c|c|c|c|c|c|c|}
\hline \multirow{2}{*}{ Time } & \multicolumn{4}{|c|}{ Real RSSI } & \multicolumn{4}{|c|}{ Predicted RSSI using CF-PSO } & \multicolumn{4}{|c|}{ Predicted RSSI using RBF } \\
\hline & AP1 & AP2 & AP3 & AP4 & AP1 & AP2 & AP3 & AP4 & AP1 & AP2 & AP3 & AP4 \\
\hline 1 & -20 & -29 & -27 & -98 & & & & & & & & \\
\hline 1.7 & & & & & -23.5 & -33.1 & -29.6 & -96.4 & -38.2 & -43.8 & -27 & -98 \\
\hline 2 & -27 & -36 & -31 & -97 & & & & & & & & \\
\hline 2.5 & & & & & -28.5 & -34.8 & -37.4 & -95.3 & -34.5 & -33 & -31 & -97 \\
\hline 3 & -30 & -37 & -40 & -96 & & & & & & & & \\
\hline 3.2 & & & & & -32.5 & -35.8 & -45.2 & -95 & -30 & -37 & -40 & -96 \\
\hline 4 & -33 & -37 & -60 & -94 & & & & & & & & \\
\hline 4.4 & & & & & -38.3 & -37.5 & -59.1 & -94.8 & -33 & -46.7 & -60 & -94 \\
\hline 5 & -38 & -38 & -68 & -93 & & & & & & & & \\
\hline 5.6 & & & & & -43.3 & -40.4 & -71.9 & -93.5 & -38 & -45.6 & -68 & -93 \\
\hline 6 & -45 & -40 & -80 & -91 & & & & & & & & \\
\hline 6.8 & & & & & -47.9 & -45.1 & -82.2 & -89.9 & -45 & -40 & -64.6 & -92.9 \\
\hline 7 & -50 & -45 & -81 & -90 & & & & & & & & \\
\hline 7.5 & & & & & -50.6 & -48.9 & -86.5 & -86.6 & -49 & -45 & -81 & -90 \\
\hline 8 & -57 & -50 & -87 & -86 & & & & & & & & \\
\hline 8.6 & & & & & -54.9 & -56.2 & -90.5 & -79.3 & -57 & -50 & -87 & -86 \\
\hline 9 & -57 & -60 & -93 & -79 & & & & & & & & \\
\hline 9.2 & & & & & -57.5 & -60.8 & -91.2 & -74.4 & -57 & -60 & -80.2 & -80.4 \\
\hline 10 & -60 & -72 & -87 & -66 & & & & & & & & \\
\hline 10.4 & & & & & -63.3 & -70.8 & -89.3 & -63 & -60 & -71.5 & -87 & -66 \\
\hline 11 & -66 & -79 & -85 & -52 & & & & & & & & \\
\hline 11.6 & & & & & -70.3 & -80.9 & -83.7 & -50.6 & -65.5 & -72 & -64.5 & -52 \\
\hline 12 & -70 & -80 & -82 & -46 & & & & & & & & \\
\hline 12.8 & & & & & -78.9 & -89.5 & -75.4 & -39 & -65.6 & -70.8 & -59.2 & -46 \\
\hline 13 & -80 & -89 & -76 & -39 & & & & & & & & \\
\hline 13.4 & & & & & -83.9 & -92.6 & -70.6 & -34.1 & -70.9 & -80 & -76 & -48.7 \\
\hline 14 & -94 & -94 & -68 & -33 & & & & & & & & \\
\hline 14.6 & & & & & -95.6 & -94.6 & -61.1 & -28.1 & -90 & -89 & -68 & -47.5 \\
\hline 15 & -98 & -95 & -56 & -26 & & & & & & & & \\
\hline
\end{tabular}

the training process was done, the reliability and overfitting of the network were verified with the test data. The overall performance of the proposed models in estimating the RSSI of four APs has been graphically depicted in Figure 7.

The real and predicted RSSI values for four APs during 15 seconds have been stated in Table 1. By looking at Table 1, it is observed that the CF-PSO model can estimate the RSSI value about $800 \mathrm{~ms}$ before the actual time.

It must be mentioned that an unsuitable selection of the initial weights may cause local minimum data. In order to prevent this unfavorable phenomenon, 30 runs for each method are applied and in each run different random values of initial weights are measured. Finally, in the RBF, the besttrained network, which has minimum MSE of validated data, is selected as the trained network. The estimation performance of CF-PSO and RBF networks is assessed by $R^{2}$ and MSE. The output values are stated in Table 2. This table shows the results of the 30 different running times with iteration $=100$.

Table 2 shows the $R^{2}$ values of all datasets for the CFPSO and RBFN. It is clear that the fit is rationally suitable for all datasets with $R$ values of 1 for the CF-PSO. The RBFN was found to be sufficient for estimation of the RSSI, whereas the CF-PSO model showed a significantly high degree of accuracy in the estimation of $R^{2}$ between 0.98 and 0.993 . In addition, the root of the MSE found that the smaller the RMSE of the test dataset, the higher the predictive quality. The assessment of the CF-PSO and RBF network models shows the suitable predictive capabilities of the CF-PSO model.

\section{Network Selection Using the Multiobjective Particle Swarm Optimization (MOPSO)}

Given the complexity of different access network technologies, an AI-based architecture would provide an efficient solution with high accuracy, stability, and faster convergence for vertical handover in heterogeneous wireless environments. One of the most important aspects of modern communications deals with access to wireless networks by mobile devices, looking for a good quality of service under the user's preferences. Nevertheless, a mobile terminal can discover 
TABLE 2: $R^{2}$ and MSE values.

\begin{tabular}{|c|c|c|c|c|c|c|c|c|c|c|c|c|c|c|c|c|}
\hline \multirow{3}{*}{ Run number } & \multicolumn{8}{|c|}{ CF-PSO } & \multicolumn{8}{|c|}{ RBFN } \\
\hline & \multicolumn{4}{|c|}{$R^{2}$} & \multicolumn{4}{|c|}{ RMSE } & \multicolumn{4}{|c|}{$R^{2}$} & \multicolumn{4}{|c|}{ RMSE } \\
\hline & AP1 & AP2 & AP3 & AP4 & AP1 & AP2 & AP3 & AP4 & AP1 & AP2 & AP3 & AP4 & AP1 & AP2 & AP3 & $\mathrm{AP} 4$ \\
\hline 1 & 0.99 & 0.98 & 0.99 & 0.99 & 0.69 & 0.78 & 0.69 & 0.63 & 0.93 & 0.72 & 0.78 & 0.72 & 1.36 & 1.98 & 1.98 & 1.98 \\
\hline 2 & 0.98 & 0.98 & 0.98 & 0.99 & 0.78 & 0.78 & 0.78 & 0.69 & 0.91 & 0.72 & 0.66 & 0.82 & 0.98 & 1.98 & 1.56 & 1.90 \\
\hline 3 & 0.97 & 0.98 & 0.99 & 0.99 & 0.79 & 0.59 & 0.69 & 0.69 & 0.80 & 0.69 & 0.67 & 0.72 & 0.90 & 1.02 & 0.98 & 1.02 \\
\hline 4 & 0.99 & 0.99 & 0.99 & 0.99 & 0.69 & 0.69 & 0.69 & 0.69 & 0.92 & 0.72 & 0.68 & 0.80 & 0.98 & 1.98 & 1.43 & 1.02 \\
\hline 5 & 0.99 & 0.98 & 0.98 & 0.99 & 0.69 & 0.79 & 0.59 & 0.59 & 0.88 & 0.70 & 0.78 & 0.86 & 0.99 & 1.36 & 1.98 & 1.98 \\
\hline 6 & 0.97 & 0.98 & 0.99 & 0.99 & 0.79 & 0.69 & 0.69 & 0.69 & 0.86 & 0.70 & 0.64 & 0.82 & 1.02 & 0.98 & 1.99 & 1.98 \\
\hline 7 & 0.99 & 0.98 & 0.99 & 0.99 & 0.69 & 0.69 & 0.69 & 0.69 & 0.93 & 0.68 & 0.77 & 0.82 & 1.36 & 1.36 & 1.36 & 0.98 \\
\hline 8 & 0.99 & 0.98 & 0.98 & 0.99 & 0.69 & 0.59 & 0.59 & 0.69 & 0.93 & 0.72 & 0.68 & 0.74 & 1.36 & 1.98 & 0.98 & 0.99 \\
\hline 9 & 0.97 & 0.99 & 0.99 & 0.98 & 0.79 & 0.59 & 0.69 & 0.89 & 0.91 & 0.72 & 0.76 & 0.74 & 0.98 & 1.98 & 1.43 & 1.02 \\
\hline 10 & 0.99 & 0.98 & 0.99 & 0.99 & 0.69 & 0.69 & 0.69 & 0.69 & 0.90 & 0.69 & 0.67 & 0.72 & 1.43 & 1.33 & 1.36 & 1.36 \\
\hline 11 & 0.99 & 0.98 & 0.99 & 0.99 & 0.69 & 0.69 & 0.69 & 0.69 & 0.72 & 0.72 & 0.68 & 0.70 & 1.35 & 1.98 & 1.36 & 1.98 \\
\hline 12 & 0.99 & 0.98 & 0.99 & 0.99 & 0.69 & 0.69 & 0.69 & 0.69 & 0.98 & 0.70 & 0.68 & 0.76 & 0.99 & 1.33 & 1.89 & 1.98 \\
\hline 13 & 0.99 & 0.99 & 0.99 & 0.98 & 0.59 & 0.69 & 0.59 & 0.80 & 0.96 & 0.70 & 0.64 & 0.72 & 1.33 & 1.36 & 0.98 & 1.89 \\
\hline 14 & 0.99 & 0.99 & 0.99 & 0.99 & 0.69 & 0.69 & 0.69 & 0.59 & 0.93 & 0.68 & 0.67 & 0.72 & 1.36 & 0.98 & 1.36 & 1.36 \\
\hline 15 & 0.99 & 0.98 & 0.99 & 0.98 & 0.69 & 0.69 & 0.69 & 0.79 & 0.93 & 0.72 & 0.58 & 0.72 & 1.36 & 1.98 & 0.98 & 1.36 \\
\hline 16 & 0.99 & 0.98 & 0.99 & 0.99 & 0.58 & 0.69 & 0.79 & 0.69 & 0.93 & 0.72 & 0.56 & 0.83 & 1.36 & 1.98 & 0.99 & 1.89 \\
\hline 17 & 0.99 & 0.98 & 0.99 & 0.99 & 0.69 & 0.59 & 0.69 & 0.69 & 0.90 & 0.69 & 0.85 & 0.82 & 1.89 & 1.89 & 1.02 & 0.98 \\
\hline 18 & 0.98 & 0.99 & 0.99 & 0.97 & 0.78 & 0.69 & 0.69 & 0.79 & 0.82 & 0.72 & 0.58 & 0.80 & 0.98 & 1.98 & 1.36 & 1.89 \\
\hline 19 & 0.99 & 0.99 & 0.97 & 0.99 & 0.69 & 0.69 & 0.69 & 0.69 & 0.88 & 0.70 & 0.58 & 0.86 & 0.99 & 1.36 & 1.36 & 0.98 \\
\hline 20 & 0.96 & 0.98 & 0.99 & 0.99 & 0.80 & 0.69 & 0.79 & 0.59 & 0.86 & 0.70 & 0.54 & 0.87 & 1.02 & 1.36 & 1.98 & 1.36 \\
\hline 21 & 0.99 & 0.98 & 0.99 & 0.99 & 0.69 & 0.69 & 0.69 & 0.69 & 0.93 & 0.68 & 0.57 & 0.82 & 1.36 & 0.98 & 1.98 & 0.98 \\
\hline 22 & 0.98 & 0.99 & 0.99 & 0.99 & 0.69 & 0.69 & 0.59 & 0.69 & 0.93 & 0.72 & 0.58 & 0.72 & 1.36 & 1.98 & 1.89 & 1.98 \\
\hline 23 & 0.99 & 0.99 & 0.99 & 0.98 & 0.69 & 0.69 & 0.69 & 0.79 & 0.91 & 0.73 & 0.56 & 0.87 & 0.98 & 0.98 & 0.98 & 1.98 \\
\hline 24 & 0.98 & 0.99 & 0.99 & 0.99 & 0.78 & 0.59 & 0.69 & 0.69 & 0.90 & 0.79 & 0.57 & 0.82 & 1.89 & 1.43 & 1.36 & 1.89 \\
\hline 25 & 0.97 & 0.98 & 0.99 & 0.99 & 0.79 & 0.69 & 0.69 & 0.79 & 0.91 & 0.72 & 0.58 & 0.80 & 0.98 & 1.98 & 0.98 & 1.89 \\
\hline 26 & 0.90 & 0.98 & 0.99 & 0.98 & 0.88 & 0.69 & 0.69 & 0.79 & 0.93 & 0.70 & 0.58 & 0.87 & 1.36 & 0.98 & 1.35 & 0.98 \\
\hline 27 & 0.99 & 0.99 & 0.97 & 0.99 & 0.69 & 0.79 & 0.79 & 0.69 & 0.92 & 0.70 & 0.54 & 0.88 & 0.98 & 1.36 & 0.98 & 1.36 \\
\hline 28 & 0.98 & 0.99 & 0.99 & 0.99 & 0.69 & 0.69 & 0.69 & 0.59 & 0.97 & 0.70 & 0.47 & 0.88 & 1.35 & 0.98 & 0.99 & 0.98 \\
\hline 29 & 0.99 & 0.99 & 0.99 & 0.98 & 0.69 & 0.69 & 0.69 & 0.79 & 0.95 & 0.72 & 0.58 & 0.82 & 1.01 & 1.98 & 1.02 & 1.35 \\
\hline 30 & 0.98 & 0.98 & 0.99 & 0.99 & 0.69 & 0.69 & 0.69 & 0.69 & 0.91 & 0.72 & 0.56 & 0.89 & 0.98 & 1.98 & 1.36 & 1.89 \\
\hline
\end{tabular}

more than one network of different technology along its route in heterogeneous scenarios that are capable of connecting to other wireless access points according to their quality of service values. The characteristics of the current mobile devices are designed to use fast and efficient algorithms that provide solutions close to real-time systems. These constraints have moved us to develop intelligent algorithms that avoid the slow and massive computations associated with direct search techniques, thus reducing the computation time.

Initially, the RSSI is used to identify the existence of wireless networks. In heterogeneous wireless environments when the mobile device senses more than one wireless network at the same time, the network selection with the best QoS becomes the main problem. In the proposed model, in the scanning phase, first, the physical layer metric (RSSI) of the available networks in the heterogeneous environments that perform an intelligence prediction is observed.

For this purpose, the CF-PSO model has been designed in this paper as a novel prediction model to construct a mathematical function, which has the best fit to a series of data points for RSSI value in UMTS and WLAN networks. In the scanning phase, the received signal strength indicator parameter is measured as the main input to the CF-PSO model to predict the received signal strength value for the four access points and to select the best AP among them using the MOPSO algorithm in an intelligent manner.

The proposed network selection model evaluates a set of cellular networks in the heterogeneous environments. The cost function measures the quality of each network at 
every location while moving from one network to another. In the vertical handover, the cost function can measure the cost utilized by handing off to a particular network. It is calculated for each network $n$, which covers the service area of a user. The wireless network technology has the ability to support high-rate data services with high spectrum efficiency. In the heterogeneous wireless networks, streaming of multimedia data over heterogeneous wireless networks has increased dramatically and video streaming services have become more popular among different types of multimedia services, such as video conferencing and voice over internet protocol. Consideration of video streaming on vertical handover where the capacity of the link is not stable is vital because the network status has an effect on the quality of video streaming. During the vertical handover, the bandwidth changes suddenly and also the handover latency leads to packet loss and disconnection of the video. In this section, we explain the network selection during the vertical handover in heterogeneous wireless networks to select the best network to offer seamless video streaming. We can see that the transferring rate and the quality of transmitted data are strongly related to the bandwidth variation.

The proposed network selection scheme presents a multiobjective decision-making (MODM) function that evaluates different cellular networks in order to select the best one in the heterogeneous networks. This function is defined by using physical layer metrics such as RSS, ABR, bit error rate (BER), SNR, and throughput of networks in heterogeneous environments.

In the next stage, the ranking of the available networks is performed based on the interface priority scores and the application priority scores assigned in stage 1 . Consider a set of candidate networks $S=\left\{s_{1}, s_{2}, \ldots, s_{N}\right\}$ and a set of quality of service factors $Q=\left\{q_{1}, q_{2}, \ldots, q_{M}\right\}$, where $N$ is the number of candidate networks and $M$ is the number of quality of service factors. In addition, each QoS factor is considered to have its own weight and this weight shows the effect of the factor on the network or user. Thus, each network's cost function can be calculated using (8), where $W_{N}$ is calculated using the MOPSO (multiobjective particle swarm optimization) MOPSO [24]. It is selected based on its ability to change its weighting among each factor according to network conditions and user preferences. Therefore,

$$
C_{N}=W_{\text {Interface }} \times \sum_{j=1}^{M} q_{j} \times W_{j}
$$

It is known that various types of services need various patterns of reliability, latency, and data rate. Therefore, we consider the service type as the main metric. In terms of network conditions, network-related parameters such as available bandwidth and network latency must be measured for efficient network usage.

In addition, the use of network information in the selection for handover can be useful for load balancing among various types of networks. Moreover, to maintain the system's performance, a range of parameters can be used in the handover decision, such as the BER. The mobile terminal condition also includes dynamic factors such as velocity, moving pattern, moving histories, and location information.

QoS is another important parameter that ensures user satisfaction to the fullest extent. The user can establish preferences for the different QoS parameters depending on the services required. The experimentation is more realistic considering different sets of the user's preferences, where, more importantly, the QoS parameters have higher values of weights. It must be mentioned that dynamic factors such as available bandwidth, velocity, and network latency have different values in various conditions. The set of significant parameters is as follows: RSS, ABR, BER, SNR, and throughput $(T)$ which is shown by vector $\mathbf{Q}_{\mathbf{N}}$ in

$$
\mathbf{Q}_{\mathbf{N}}=[\operatorname{RSS} \text { ABR BER SNR T]. }
$$

At the end, based on (8), the cost values of each user's requested service from network $n$ can be computed. The computation time constraint of MOPSO algorithm is relatively low and eventually conforms to the multimedia time requirement for reliable communication over wireless networks. Several algorithms related to the MOPSO have been proposed in the literature [25]. When using the MOPSO, it is possible for the degree of the velocities to become very large. Two techniques were advanced to control the increase of velocities. The first is to modify the inertia factor in a dynamic manner and the second is to use the constriction coefficient. In order to improve the inertia and convergence of the particle over time, a variant of MOPSO called the Modified MOPSO [24] is used, where the inertia factor $(\omega)$ and constriction coefficient $(\delta)$ have been introduced in the velocity update as shown in (1), respectively. The network having the lowest cost function is selected as the "always best connected" network or optimum access network in heterogeneous environments. Based on the MOPSO, a new handover decision algorithm is proposed to seek the best network to connect the mobile node to the access network. The proposed model has a cost function to measure the quality of networks at each situation. Our scheme predicted the RSSI each time to enhance the selection, and the outputs of the prediction unit should be sent to the selection unit. Since the selection of the best network is a multiobjective problem, hence the MOPSO can be efficient because access network selection has conflicting objectives in terms of minimization and maximization. For example, the vertical handover decision algorithm should select the access network with high RSSI, ABR, SNR, and throughput, and this algorithm also needs to select the best network with low BER, handover rate, and outage probability. The operation of our strategy is shown in Pseudocode 1.

\section{Computational Experiments on the Multiobjective Particle Swarm Optimization (MOPSO)}

In Section 3.3, the efficacy of the CF-PSO to predict the received signal strength value for the four access points in the neighborhood is shown. We explain the MOPSO as a novel model, the best choice for the candidate access point, and as a smart approach in the designed scenario. In the 
$\% \%$ MOPSO Parameters

MaxIt $=200$;

$\mathrm{nPop}=50$;

$\mathrm{nRep}=50$;

$\mathrm{w}=0.5$;

wdamp $=0.99$;

$\mathrm{c} 1=1$;

$\mathrm{c} 2=2$;

nGrid $=5$;

alpha $=0.1$;

beta $=2$;

gamma $=2$;

$\mathrm{mu}=0.1$;

$\% \%$ Initialization

pop=repmat (empty_particle,nPop,1) ;

for $i=1: n P o p$

pop (i).Position=unifrnd (VarMin, VarMax, VarSize);

pop (i).Velocity=zeros (VarSize);

pop (i). Cost=CostFunction (pop (i).Position);

\% Update Personal Best

pop (i). Best.Position=pop (i).Position;

pop (i). Best. Cost=pop (i). Cost;

end

\% Determine Domination

pop=DetermineDomination (pop);

rep $=\operatorname{pop}(\sim$ [pop. IsDominated]);

Grid=CreateGrid (rep,nGrid, alpha);

for $i=1$ : numel ( $r e p$ )

end

$\operatorname{rep}(i)=$ FindGridIndex $(\operatorname{rep}(i), \operatorname{Grid})$;

$\% \%$ MOPSO Main Loop

for it $=1$ : MaxIt

for $i=1: n P o p$

leader=SelectLeader (rep, beta);

pop (i).Velocity $=\mathrm{w} * \operatorname{pop}(i) \cdot$ Velocity ...

$+c 1 * r a n d(\operatorname{VarSize}) . *($ pop(i).Best.Position-pop(i).Position) ...

$+c 2 * r a n d$ (VarSize).*(leader.Position-pop(i).Position);

pop (i). Position $=$ pop (i). Position + pop (i).Velocity;

pop (i). Cost $=$ CostFunction (pop(i).Position);

$\%$ Apply Mutation

$\mathrm{pm}=(1-(i t-1) /(\operatorname{MaxIt}-1))^{\wedge}(1 / \mathrm{mu})$;

NewSol .Position=Mutate (pop(i).Position, pm, VarMin, VarMax);

NewSol. Cost=CostFunction (NewSol.Position);

rep $=[\mathrm{rep} ; \operatorname{pop}(\sim[\mathrm{pop}$. IsDominated] $)]$; \% Add Non-Dominated Particles to REPOSITORY rep=DetermineDomination ( $\mathrm{rep}$ ); \% Determine Domination of New Resository Members rep $=r e p(\sim$ rep. IsDominated]); \% Keep only Non-Dminated Memebrs in the Repository Grid=CreateGrid (rep,nGrid,alpha); \% Update Grid

for $i=1$ : numel (rep) \% Update Grid Indices

$\operatorname{rep}(i)=$ FindGridIndex $(\operatorname{rep}(i), \operatorname{Grid})$;

end

if numel (rep) $>$ nRep $\quad \%$ Check if Repository is Full

Extra=numel (rep)-nRep;

for $e=1$ : Extra

rep=DeleteOneRepMemebr (rep, gamma);

end

end

Pseudocode 1: Network selection by MOPSO pseudo-code. 


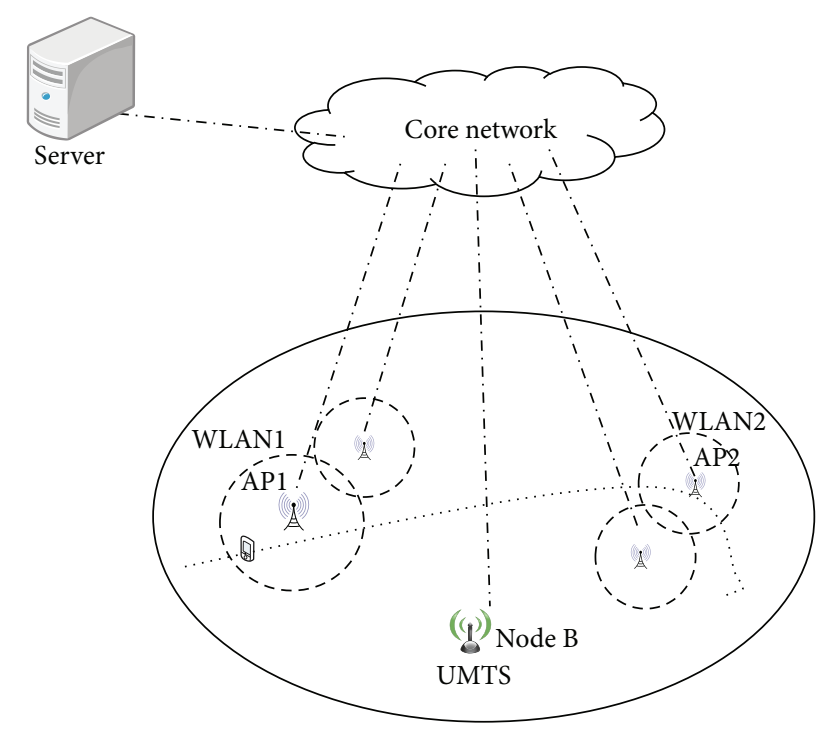

FIGURE 8: Simulation scenario.

MOPSO algorithm, we have performed simulations to show the feasibility of the proposed scheme using the simulation software, QualNet 7.0. This experiment is for the case of a vertical handover between a $3 \mathrm{G}$ network and WLANs. The scenario simulated in the QualNet is composed of the UMTS BS and IEEE 802.11 APs. The WLAN is based on the IEEE $802.11 \mathrm{~b}$ standard, and the physical data rate is $11 \mathrm{Mbps}$. The capacity of a 3G Universal Mobile Telecommunications System is $384 \mathrm{kbps}$. All links except for the wireless links have a capacity of $100 \mathrm{Mbps}$ each. Ad hoc on-demand distance vector (AODV) protocol is used as the reactive routing protocol [26]. This protocol offers quick convergence when the ad hoc network topology changes (typically, when a node moves in the network).

As shown in Figure 8, the mobile node can be at a fixed time in the coverage area of the UMTS. Nevertheless, due to movement, it can travel into the areas that cover more than one access network, that is, simultaneously within the coverage areas of, for example, a UMTS BS and an IEEE 802.11 AP. Multiple IEEE 802.11 WLAN coverage areas are usually comprised within a UMTS coverage area. Since the WLAN1 has a lower coverage range when the mobile user is moving out of a WLAN1 area, the existence of an accurate and timely handoff decision to maintain the connectivity before the loss of the WLAN access is necessary. Subsequently, the user could move into the regions covered by a UMTS network and then the user could move into a WLAN2 area to achieve a higher QoS at the lowest cost. Therefore, the user changes the connection to the WLAN2. The mobile node associated with the UMTS or WLANs monitors and measures $D_{\mathrm{RSS}}$ which is the diversity of the received signal strength between the networks of the nearby WLANs/UMTS to check whether an access network with high data rate is offered.

The performance of the proposed algorithm has been assessed in a scenario when the mobile node moves with a constant speed along a straight line path from the area covered by WLAN1 to the one covered by UMTS and then roams to the area covered by WLAN2. Clearly, with the increase of distance, the average of RSS, ABR, SNR, and throughput will be reduced and the BER will also be increased. By calculating the cost value based on the number of iterations, an optimal network can be selected as presented in Figure 9.

The MOPSO algorithm using the CF-PSO prediction is compared to the RBF network based on the number of vertical handoffs. The scenario of a heterogeneous wireless network consisting of WLANs and Universal Mobile Telecommunications System (UMTS)/B3G is demonstrated in Figure 8. In the simulations, the evaluated heterogeneous wireless networks contain 4 to 10 mobile terminals and 10 WLANs. The mobility of random way point is adopted for each mobile node with random direction and random velocity from 1 to $25 \mathrm{~m} / \mathrm{s}$, the number of UMTSs equals 1 , arrival rate of Poisson distribution is 6 to 16 , and the bandwidths of B3G/UMTS and WLAN are $384 \mathrm{~kb} / \mathrm{s}$ and $54 \mathrm{Mb} / \mathrm{s}$, respectively. The topology covers an area of $2000 \mathrm{~m}$ in length and $2000 \mathrm{~m}$ in width. The simulation considers two classes of traffic, that is, constant bit rate (CBR) and variable bit rate (VBR). Bursty applications produce VBR (variable bit rate) traffic streams, while constant applications produce CBR traffic streams. The CBR traffic stream is easy to model and to predict its impact on the performance of the network. Data rates of CBR of B3G and WLAN are 50 and 200 (Kbps) and low and high level data rates of VBR for B3G and WLAN are 10 (Kbps) and $1.6(\mathrm{Mbps})$, respectively [27].

Figure 10 shows the evaluation of the proposed approach by comparing the number of vertical handoffs. Clearly, the proposed prediction approach considerably improves the number of vertical handoffs. The $95 \%$ confidence intervals of the simulation results in Figure 10 are created from 30 independent runs. The number of vertical handoffs of all the approaches increases when the arrival rate increases. The proposed prediction model, the CF-PSO approach, results in the fewest vertical handoffs. Consequently, the CF-PSO approach outperforms other approaches in the number of vertical handoffs in heterogeneous wireless networks.

Figure 11 shows the variation of state between the cellular heterogeneous networks with respect to the distance based on the proposed algorithm. Clearly, when the distance increases, the cost value increases in the cellular networks. The effect of increasing the number of iterations on the cost value has been examined to find the number of iterations essential to reach optima. Iterations are different from 10 to 100 based on the fact that no significant variation was detected after 100 successive iterations for WLANs and UMTS cellular networks, respectively.

\section{Conclusion}

We have proposed a new MOPSO-based vertical handover decision algorithm. Our goal was to achieve an optimized MOPSO in a small cost function, and for this reason, the MOPSO rules were proposed for the heterogeneous wireless networks. The CF-PSO prediction model is able to reduce 


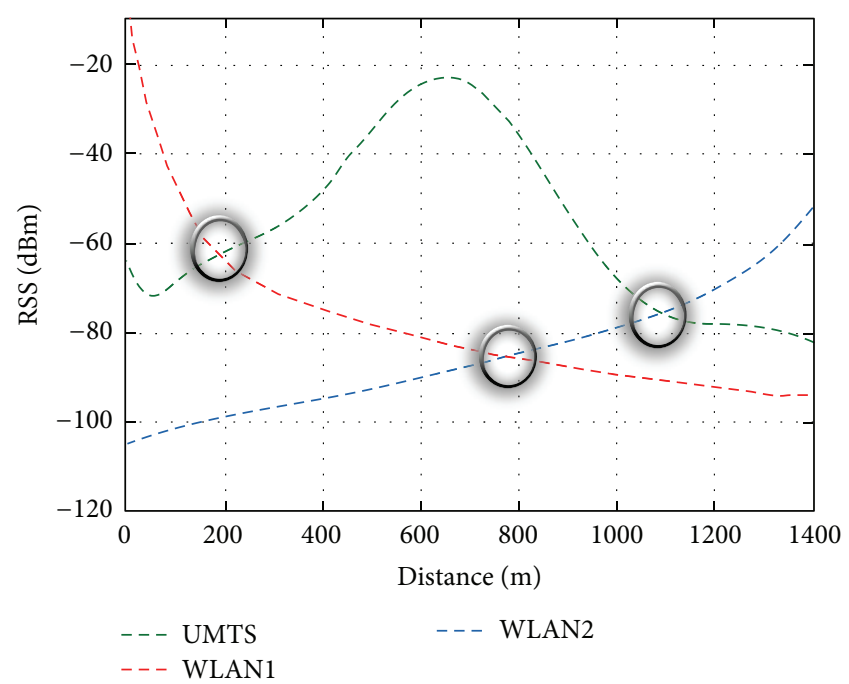

(a)

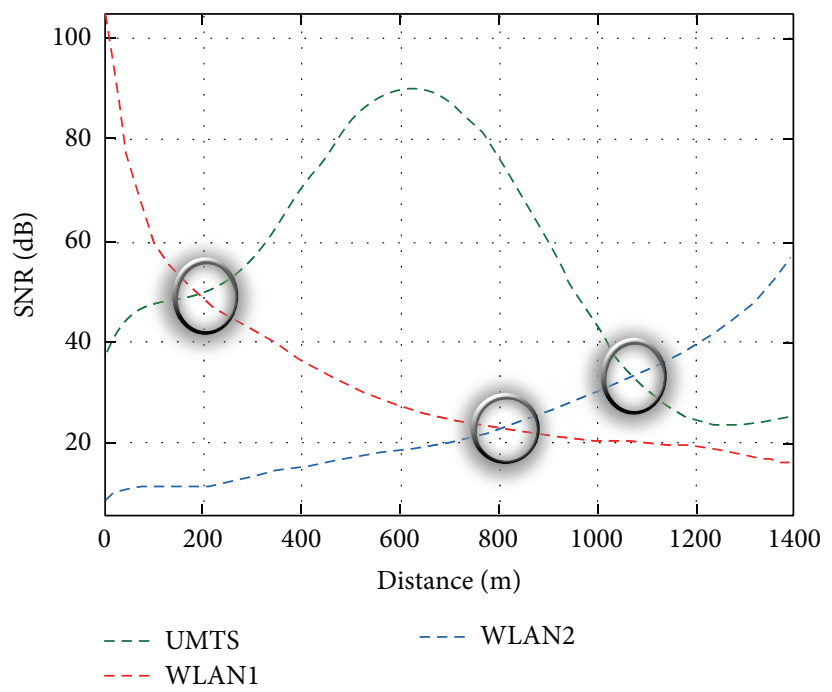

(c)

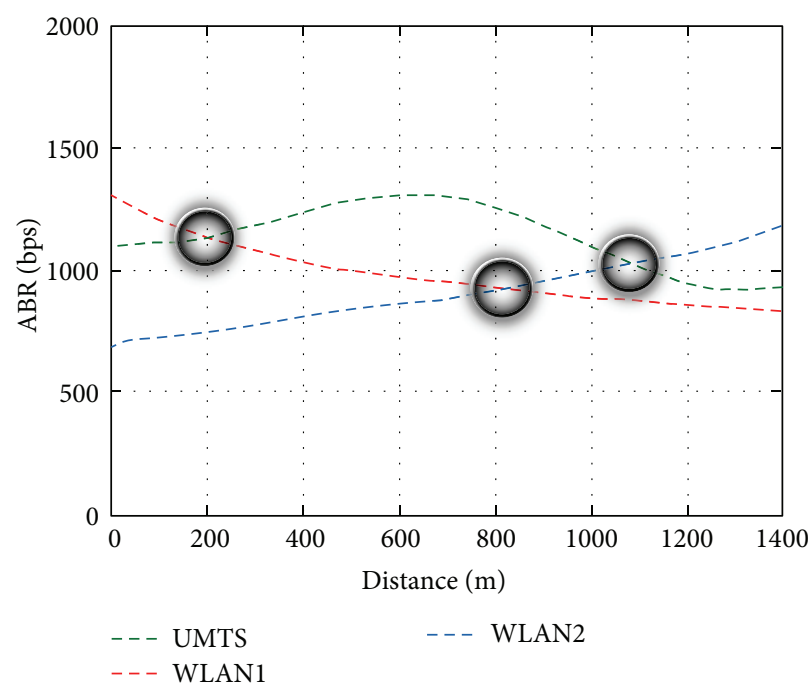

(b)

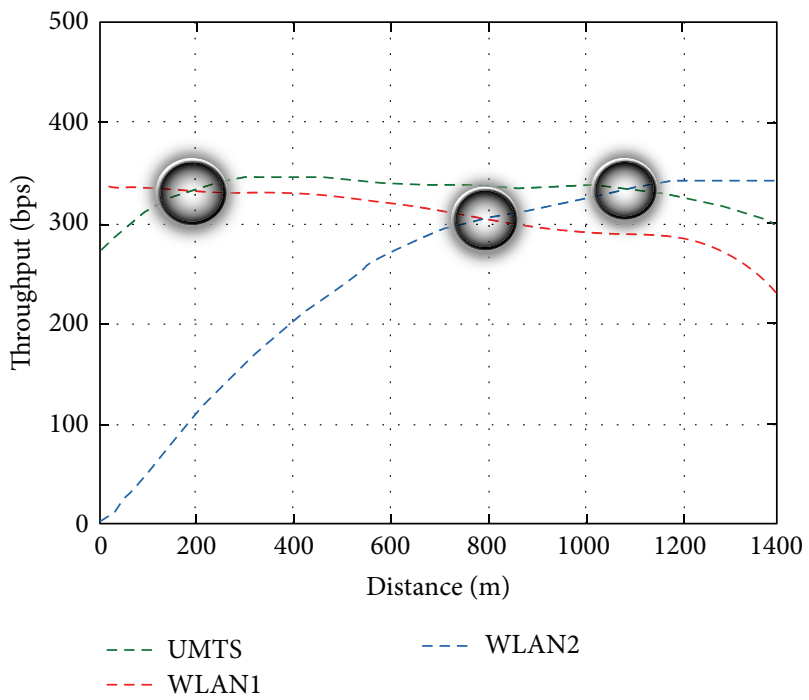

(d)

FIGURE 9: (a) Average RSS versus distance, (b) ABR versus distance, (c) SNR versus distance, and (d) throughput versus distance in cellular heterogeneous environment of WLANs and UMTS, where ABR: ABR $=$ bandwidth $\times \log _{2}(1+\mathrm{SNR}) i=1,2,3[22]$ and SNR: SNR $i=E_{b} / N_{o}$ [23]; $E_{b}$ is the received energy per bit and $N_{o}$ is the noise power of the channel.

the number of unnecessary handovers and avoid the "PingPong" effect in the heterogeneous wireless environments. Our novel section scheme was aimed at making mobile nodes smart enough to be able to autonomously determine the best network using data prediction. The experimental results show that, in the prediction of the received signal strength indicator parameter, the model based on CF-PSO is more effective than the RBF neural network model. The selection decision function is defined as a cost function consisting of five parameters, namely, RSS, ABR, BER, SNR, and throughput $(T)$. The proposed approach performed well with several test problems in terms of the number of cost function evaluations required, the quality of the solutions found, the rate of successful minimizations, and the average cost functions. Several experiments were performed for the optimization of the vertical handover decision-making algorithms in an intelligent manner in the heterogeneous wireless networks. The MOPSO-VHO has a low cost function compared to previous works. Simulation results indicated that our proposed vertical handover decision algorithm was able to minimize the cost function, reduce the number of unnecessary handovers, avoid the "Ping-Pong" effect, and select the best access network that is optimized to network conditions, quality of service requirements, mobile terminal conditions, and user preferences.

\section{Conflict of Interests}

The authors declare that there is no conflict of interests regarding the publication of this paper. 


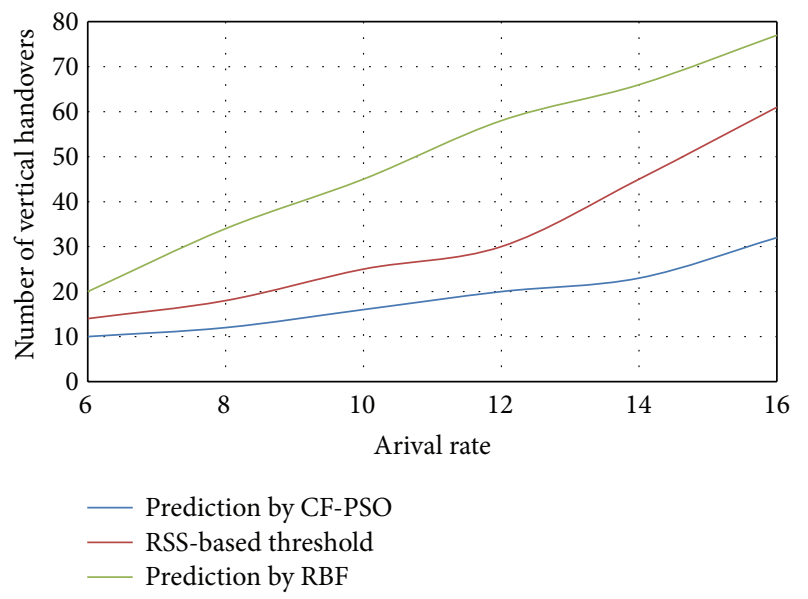

Figure 10: Comparing the number of vertical handoffs under various arrival rates.

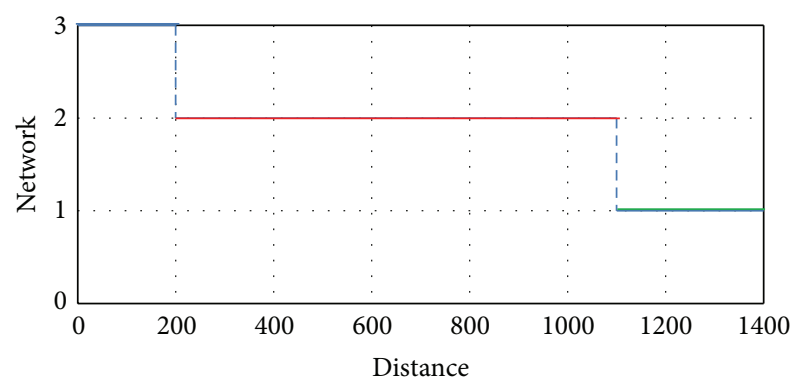

FIGURE 11: Network selection between WLANs and UMTS based on the proposed algorithm.

\section{Acknowledgments}

The authors would like to thank the Ministry of Education, Malaysia, for funding this research project through a Research University Grant of Universiti Teknologi Malaysia (UTM), Malaysian Japan International Institute of Technology (MJITT), and i-Kohza Computer System and Network (CSN). Also, the authors thank University of Malaya for the financial support (BKP Grant BK060-2014) and facilities to carry out the work.

\section{References}

[1] L. Giupponi, R. Agusti, J. Pérez-Romero, and O. Sallent, "A novel joint radio resource management approach with reinforcement learning mechanisms," in Proceedings of the 24th IEEE International Performance, Computing, and Communications Conference (IPCCC '05), pp. 621-626, IEEE, Phoenix, Ariz, USA, April 2005.

[2] A. Wilson, A. Lenaghan, and R. Malyan, "Optimising wireless access network selection to maintain QoS in heterogeneous wireless environments," in Proceedings of the 8th International Symposium on Wireless Personal Multimedia Communications (WPMC '05), Aalborg, Denmark, September 2005.
[3] A. Ahmed, L. M. Boulahia, and D. Gaïti, "Enabling vertical handover decisions in heterogeneous wireless networks: a stateof-the-art and a classification," IEEE Communications Surveys \& Tutorials, vol. 16, no. 2, pp. 776-811, 2014.

[4] S. Paul, J. Pan, and R. Jain, "Architectures for the future networks and the next generation internet: a survey," Computer Communications, vol. 34, no. 1, pp. 2-42, 2011.

[5] P. TalebiFard, T. Wong, and V. C. M. Leung, "Access and service convergence over the mobile internet-a survey," Computer Networks, vol. 54, no. 4, pp. 545-557, 2010.

[6] Z. Movahedi, M. Ayari, R. Langar, and G. Pujolle, "A survey of autonomic network architectures and evaluation criteria," IEEE Communications Surveys and Tutorials, vol. 14, no. 2, pp. 464490, 2012.

[7] V. Rakovic and L. Gavrilovska, "Novel RAT selection mechanism based on Hopfield neural networks," in Paper Presented at the International Congress on Ultra Modern Telecommunications and Control Systems and Workshops (ICUMT '10), pp. 210-217, Moscow, Russia, October 2010.

[8] A. Çalhan and C. Çeken, "Artificial neural network based vertical handoff algorithm for reducing handoff latency," Wireless Personal Communications, vol. 71, no. 4, pp. 2399-2415, 2013.

[9] A. Çalhan and C. Çeken, "Case study on handoff strategies for wireless overlay networks," Computer Standards and Interfaces, vol. 35, no. 1, pp. 170-178, 2013.

[10] N. Wang, W. Shi, S. Fan, and S. Liu, "PSO-FNN-based vertical handoff decision algorithm in heterogeneous wireless networks," Procedia Environmental Sciences, vol. 11, part A, pp. 55-62, 2011.

[11] X. Liu and L.-G. Jiang, "A novel vertical handoff algorithm based on fuzzy logic in aid of grey prediction theory in wireless heterogeneous networks," Journal of Shanghai Jiaotong University (Science), vol. 17, no. 1, pp. 25-30, 2012.

[12] A. Singhrova and N. Prakash, "Vertical handoff decision algorithm for improved quality of service in heterogeneous wireless networks," IET Communications, vol. 6, no. 2, pp. 211-223, 2012.

[13] K. Pahlavan, P. Krishnamurthy, A. Hatami et al., "Handoff in hybrid mobile data networks," IEEE Personal Communications, vol. 7, no. 2, pp. 34-47, 2000.

[14] Z. Altman, S. Sallem, R. Nasri, B. Sayrac, and M. Clerc, "Particle swarm optimization for mobility load balancing SON in LTE networks," in Proceedings of the IEEE Wireless Communications and Networking Conference Workshops (WCNCW'14), pp. 172177, IEEE, Istanbul, Turkey, April 2014.

[15] X.-W. Wang, P.-Y. Qin, M. Huang, and H. Cheng, "Niche PSO based QoS handoff decision scheme with ABC supported," in Proceedings of the IEEE International Conference on Intelligent Computing and Intelligent Systems (ICIS '09), pp. 423-427, Shanghai, China, November 2009.

[16] S. Venkatachalaiah, R. J. Harris, and J. Murphy, "Improving handoff in wireless networks using Grey and particle swarm optimisation," in Proceedings of the 2nd International Conference on Computing, Communication and Control Technologies (CCCT '04), vol. 5, pp. 368-373, Austin, Tex, USA, August 2004.

[17] M. Rehan, M. Yousaf, A. Qayyum, and S. Malik, "A cross-layer user centric vertical handover decision approach based on MIH local triggers," in Wireless and Mobile Networking, vol. 308, pp. 359-369, Springer, Berlin, Germany, 2009.

[18] M. O. Ansong, H.-X. Yao, and J. S. Huang, "Radial and sigmoid basis function neural networks in wireless sensor routing topology control in underground mine rescue operation based 
on particle swarm optimization," International Journal of Distributed Sensor Networks, vol. 2013, Article ID 376931, 14 pages, 2013.

[19] C.-H. Kuo, T.-A. Liu, J.-H. Chen, C.-M. J. Chang, and C.J. Shieh, "Response surface methodology and artificial neural network optimized synthesis of enzymatic 2-phenylethyl acetate in a solvent-free system," Biocatalysis and Agricultural Biotechnology, vol. 3, no. 3, pp. 1-6, 2014.

[20] P. Shabanzadeh, R. Yusof, and K. Shameli, "Artificial neural network for modeling the size of silver nanoparticles' prepared in montmorillonite/starch bionanocomposites," Journal of Industrial and Engineering Chemistry, vol. 24, pp. 42-50, 2015.

[21] G. M. Foody, "Supervised image classification by MLP and RBF neural networks with and without an exhaustively defined set of classes," International Journal of Remote Sensing, vol. 25, no. 15, pp. 3091-3104, 2004.

[22] H. D. Pfister, J. B. Soriaga, and P. H. Siegel, "On the achievable information rates of finite state ISI channels," in Proceedings of the IEEE Global Telecommunications Conference (GLOBECOM '01), vol. 5, pp. 2992-2996, IEEE, San Antonio, Tex, USA, November 2001.

[23] F. R. Reza, "Optimum ranges for data transmission in mobile communications," International Journal of Scientific \& Engineering Research, vol. 3, no. 4, pp. 481-489, 2012.

[24] C. A. Coello Coello and M. S. Lechuga, "MOPSO: a proposal for multiple objective particle swarm optimization," in Proceedings of the Congress on Evolutionary Computation (CEC '02), vol. 2, pp. 1051-1056, IEEE, Honolulu, Hawaii, USA, May 2002.

[25] M. Reyes-Sierra and C. A. Coello Coello, "Multi-objective particle swarm optimizers: a survey of the state-of-the-art," International Journal of Computational Intelligence Research, vol. 2, no. 3, pp. 287-308, 2006.

[26] C. E. Perkins, E. M. Royer, S. R. Das, and M. K. Marina, "Performance comparison of two on-demand routing protocols for ad hoc networks," IEEE Personal Communications, vol. 8, no. 1, pp. 16-28, 2001.

[27] B.-J. Chang and J.-F. Chen, "Cross-layer-based adaptive vertical handoff with predictive RSS in heterogeneous wireless networks," IEEE Transactions on Vehicular Technology, vol. 57, no. 6, pp. 3679-3692, 2008. 


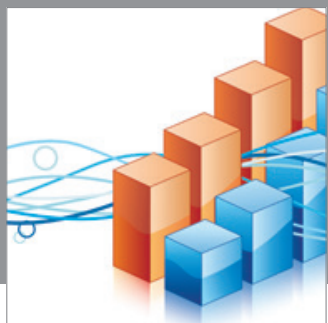

Advances in

Operations Research

mansans

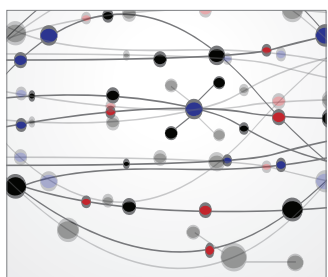

The Scientific World Journal
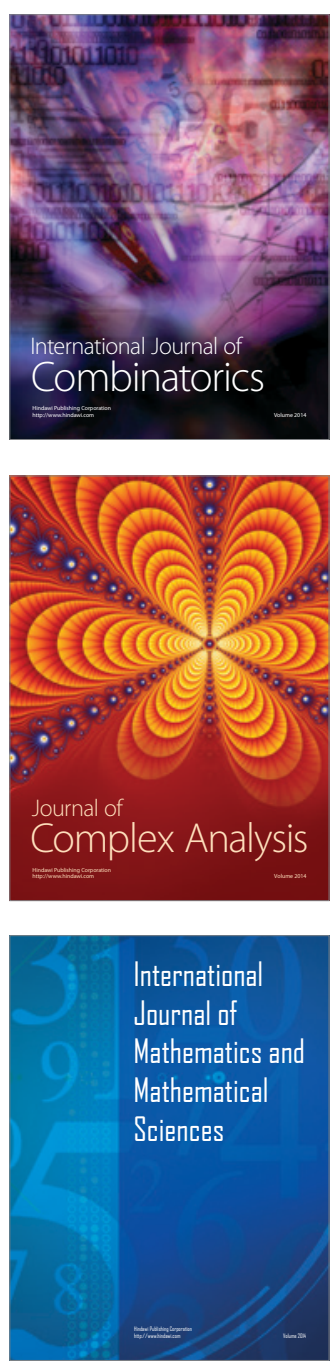
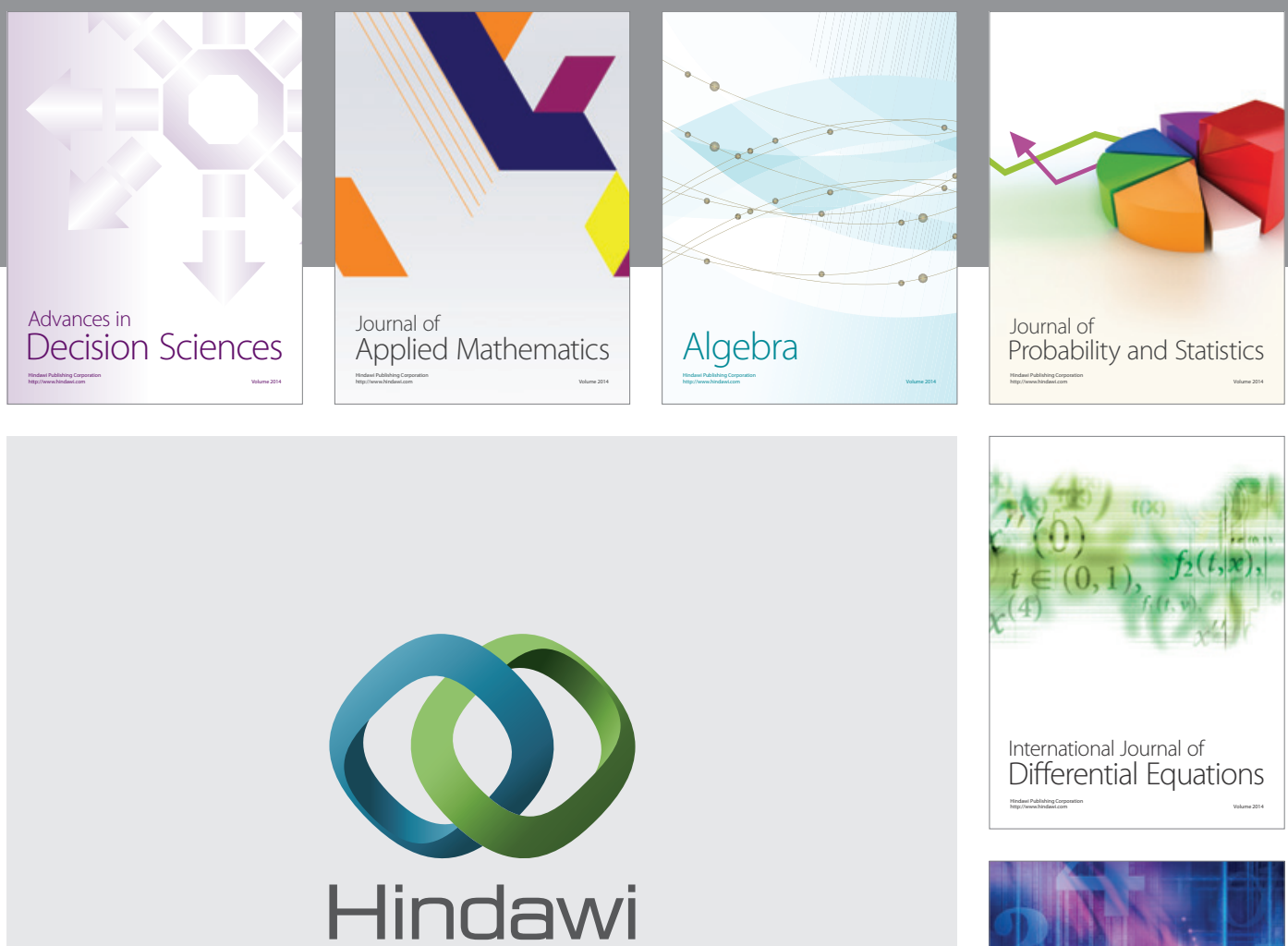

Submit your manuscripts at http://www.hindawi.com
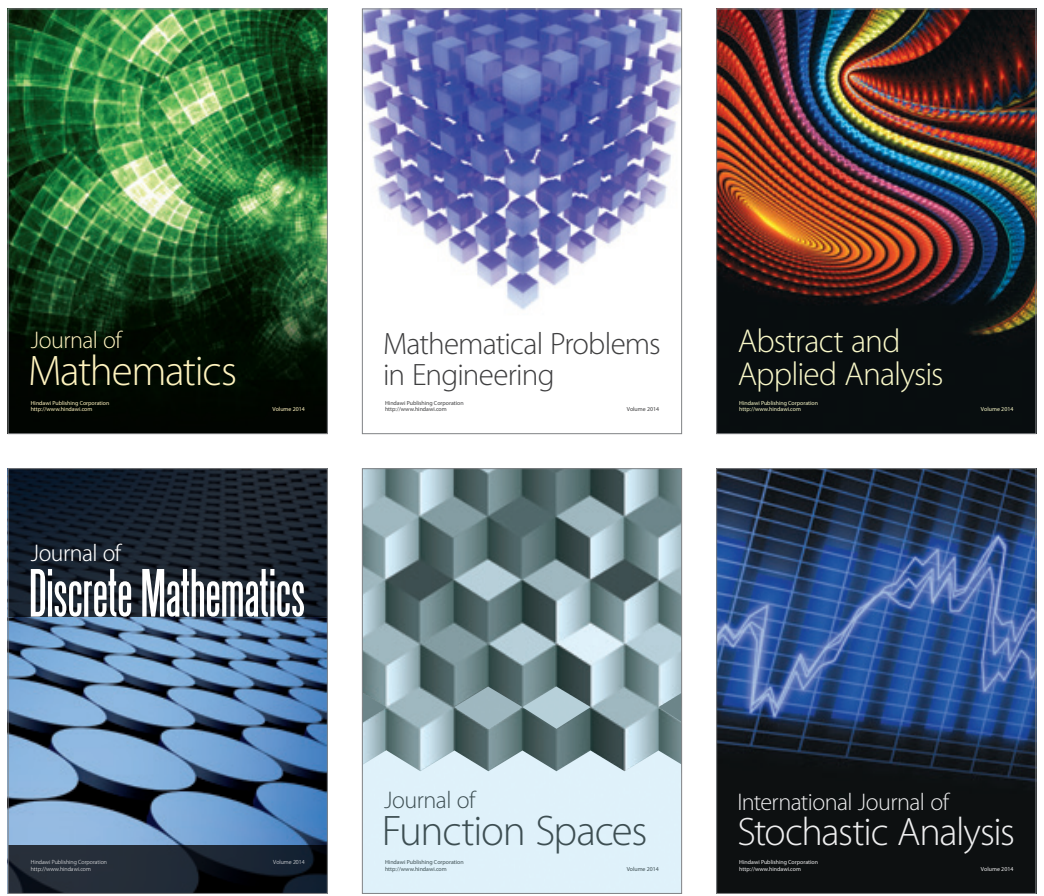

Journal of

Function Spaces

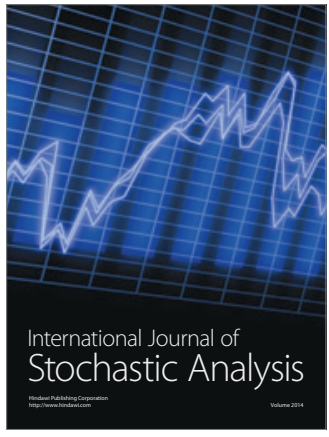

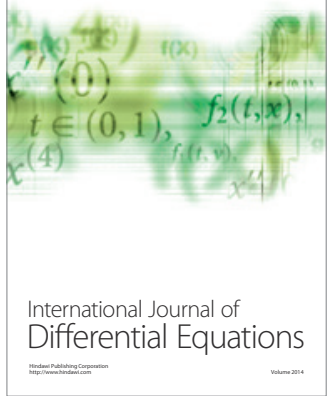
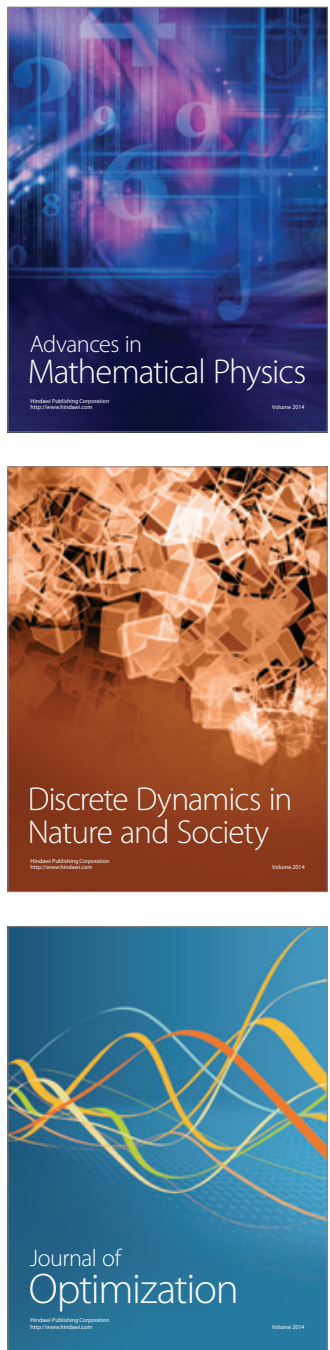